\title{
AUFSÄTZE
}

Sven Chojnacki/Fabian Namberger

\section{Die »neuen Kriege« im Spiegel postkolonialer Theorien und kritischer Friedensforschung}

\author{
Ein Plädoyer für die Befreiung von der Last der Vereinfachung
}

Mit der These der "neuen Kriege" haben Mary Kaldor und Herfried Münkler einen debattenwirksamen Begriff geliefert, auf dessen immanente methodische und theoretische Schwächen bereits aus Sicht verschiedener Wissenschaftstraditionen innerhalb und außerhalb der Friedens- und Konfliktforschung verwiesen wurde. Trotz vielfältiger Kritik mangelt es jedoch nach wie vor an einer systematischen Auseinandersetzung mit der wissenschaftichen sowie politischen Wirkungsmächtigkeit der Thesen von den »neuen Kriegen « sowie damit verbundener diskursiver Formationen (u. a. Staatszerfall, Intervention). Mit diesem Beitrag entwickeln wir entlang postkolonialer Theorieansätze alternative Deutungshorizonte, die bisherige Kritikentwürfe erweitern. Der Beitrag identifiziert das Kriegs- und Staatsverständnis der "neuen Kriege« dabei nicht nur als simplifizierenden Eurozentrismus, sondern als eine diskursive Praxis, die sowohl subalterne Identitäten in den »neuen Kriegen" erfindet und essenzialisiert als auch die Legitimation westlicher (Militär-)Interventionen begünstigt bzw. diese gar forciert - und so zur Verfestigung bestehender globaler Machtverhältnisse und Ordnungsstrukturen beiträgt.

Schlagworte: »Neue Kriege«, failed states, Intervention, Konfliktforschung, Postkoloniale Theorien, Eurozentrismus

\section{Einleitung}

Längst entzaubert, aber dennoch langlebig und in unheilvoller Komplizenschaft mit der Legitimierung militärischer Interventionspolitiken: Wer die Erwartung hatte, die These von den »neuen Kriegen « wäre nur eine Episode der Friedens- und Konfliktforschung, sieht sich getäuscht oder gar ernüchtert. Auch wenn es Vertreter_innen dieser Forschungsdisziplin eigentlich gewohnt sind, in einen Dauer-Wettstreit 
um binäre Begriffspaare zu treten (u. a. positiver vs. negativer Frieden, physische vs. strukturelle Gewalt), ist die wissenschaftliche Karriere der »neuen Kriege« mindestens verblüffend, mehr noch irritierend - und zwar sowohl aus konfliktanalytischen Gründen als auch aus einer Perspektive postkolonialer Kritik. ${ }^{1}$

Zur Erinnerung: Mary Kaldor (1999) und etwas später auch Herfried Münkler $(2002 ; 2010)$ haben den Begriff der »neuen Kriege « plakativ in die wissenschaftliche und öffentliche Debatte eingeführt, um eine bestimmte Mixtur gegenwärtiger Kriege herauszustellen und von »alten Kriegen« abzugrenzen. Dabei werden insbesondere Merkmale wie die Beteiligung nichtstaatlicher Gruppierungen in sogenannten »zerfallenden Staaten« (u. a. Warlords, Kindersoldat_innen, private Militärfirmen), die Finanzierung der Kriege und damit verbundene ökonomische Motive der »Gier« und Strukturen globalisierter Kriegswirtschaften sowie die Methoden einer »brutalisierten« und »sexualisierten « Kriegführung (Vertreibung und Vergewaltigung als Kriegswaffe) als empirische Belege für ein gewandeltes Kriegsgeschehen herangezogen und »alten« europäischen Staatenkriegen gegenübergestellt.

Verblüffend ist die Langlebigkeit des Konzepts, weil zahlreiche Analysen innerhalb der Friedens- und Konfliktforschung die binäre Gegenüberstellung von »alten « und »neuen Kriegen« entlang ihrer begriffsanalytischen und methodischen Vagheit, empirischen Ungenauigkeit und theoretischen Inkonsistenzen längst entzaubert hatten (u. a. Berdal 2011; Chojnacki 2006; Henderson/Singer 2002; Kahl/Teusch 2004; Kalyvas 2001; Melander et al. 2009; Newman 2004). So dachten zumindest viele! Zum einen dient die Folie des »Neuen« Konfliktanalysen jedoch weiterhin als Referenzpunkt für die Einordnung von Gewaltdynamiken jenseits des Staates und der Abgrenzung von »westlich«-demokratischer Kriegführung (u. a. Zangl/Zürn 2003; Heupel/Zangl 2004). ${ }^{2}$ Zum anderen haben die beiden Hauptvertreter_innen noch einmal nachgelegt: Während Münkler (2013) die »neuen Kriege« in einer Art Husarenstreich inzwischen in den Adelsstand einer »Theorie« erhoben hat, hat Kaldor (2012) in der dritten Auflage ihres Werkes »New and Old Wars. Organized Violence in a Global Era« ihre Beobachtungen auf die Kriege im Irak und in Afghanistan ausgeweitet. In einer weiteren Verteidigungsrede hat sie dann die Notwendigkeit dieser begriffsanalytischen Kategorisierung für die Weiterentwicklung von »kosmopolitischen Strategien« der Rechtsdurchsetzung noch einmal hervorgehoben und damit die wissenschaftliche Komplizenschaft mit der politischen Legitimierung

1 Die Verfasser bedanken sich für die wertvollen und konstruktiven Hinweise der anonymen Gutachter_innen, die zur Strukturierung und Schärfung der Argumentation einen wesentlichen Beitrag geleistet haben.

2 Siehe hierzu ausführlich Abschnitt 2. 
»westlicher « Interventionspraktiken erneuert: »What is still lacking in the debate is the demand for a cosmopolitan political response « (Kaldor 2013: 14).

Wenn jedoch machtformende Begriffspaare (»alte« vs. »neue Kriege«, failed states vs. starker Staat), dualistische Selbst- und Fremdbilder (»westlicher Peacebuilder « vs. »barbarischer Kämpfer« oder »islamischer Terrorist«) sowie wenig reflektierte und teilweise biologistische Argumente, die maßgeblich die Faszination des Mannes für Kriege (van Creveld 1991; zur Kritik Gantzel 2002) oder »Zonen toxischen Testosterons « (Ignatieff, zitiert in Münkler 2002: 39) zur Erklärung veränderter Kriegswirklichkeiten heranziehen, als Wegweiser öffentlicher Meinung und wissenschaftlicher Deutung oder gar für die Entwicklung von Normen zur Durchsetzung internationaler Ordnungskonzepte (siehe Kaldor 2013) dienen, droht der kritischen Friedens- und Konfliktforschung ein Kompetenzproblem. Dieses beinhaltet insbesondere die abnehmende Fähigkeit, mit alternativen und theoretisch wie empirisch differenzierten Deutungsangeboten innerhalb und außerhalb disziplinärer Grenzen Gehör zu finden. Und genau an diesem Punkt verbindet sich die Verblüffung über die Karriere der Rede von den »neuen Kriegen« mit Irritation vor allem über die offenkundige Schwäche der Friedens- und Konfliktforschung, ihrer häufig unreflektierten Verwendung in Wissenschaft und Praxis alternative Deutungsangebote entgegenzusetzen und vor allem die Potenziale vorhandener postkolonialer Kritikperspektiven dafür produktiv zu nutzen.

Um nicht missverstanden zu werden: Obwohl die These von den »neuen Kriegen« bereits aus unterschiedlichen und sogar konkurrierenden Wissenschaftstraditionen kritisch in die Zange genommen worden ist, ist das Potenzial kritischer Perspektiven bei Weitem nicht ausgereizt worden. So haben zwar sowohl politikwissenschaftlichquantitative Analysen als auch makrosoziologisch-qualitative Ansätze auf die immanenten Schwächen der methodischen und theoretischen Konsistenz der »neuen Kriege« verwiesen (vage Kriterien, fehlende intersubjektive Überprüfbarkeit, fehlende Generalisierbarkeit aufgrund schwacher empirischer Belege). Doch nach wie vor mangelt es an einer systematischen Auseinandersetzung mit der Wirkungsmächtigkeit und den Konsequenzen, die die begriffstheoretische Rahmung »neuer Kriege « und daran anschließende diskursive Formationen wie »Staatszerfall«, »transnationaler Terrorismus « und »Intervention« in Wissenschaft und Politik entfalten. Damit sollte auch bereits deutlich geworden sein, dass die hier formulierten Kritikperspektiven auf die »neuen Kriege« stellvertretend für verwandte Konzepte wie die failed-states-Debatte (siehe u. a. Goldstone 2008; Rotberg 2007; Schneckener 2005) oder die ebenfalls binär angelegte greed-and/or-grievance-Debatte stehen (Collier/Hoeffler 2004), die in den zurückliegenden Jahren nicht weniger prominent die wissenschaftlichen Auseinandersetzungen über Ursachen und 
Wandel des Krieges geprägt und Eingang in die sicherheitspolitischen Überlegungen »westlicher« Außenpolitiken gefunden haben.

Um eine produktive Auseinandersetzung anzustoßen, gehen wir in Anlehnung an Judith Butler (2009: 32) davon aus, dass der Rahmen der »neuen Kriege« nicht nur einfach bestimmte problematische materielle Aspekte des Krieges bzw. seines Wandels reflektiert, sondern sowohl einen diskursiv erzeugten politischen Richtungssinn vorgibt als auch den visuellen Rahmen für das Appellieren an moralische Pflichten prägt - und damit die politischen Interpretationen von Krieg sowie die Entscheidungen zum Krieg beeinflusst. Aus der Perspektive postkolonialer Kritiken lässt sich das Kriegs- und Staatsverständnis der »neuen Kriege« somit nicht nur als simplifizierender Eurozentrismus ${ }^{3}$ bestimmen, sondern als eine diskursive Praxis verstehen, die subalterne Identitäten in »neuen Kriegen« »erfindet « und essenzialisiert (Spivak 2008). Die damit verbundene Differenz zwischen dem »Wir« und den »Anderen« der "neuen Kriege« wird so zum Bestandteil einer politischen Selbstrepräsentation »des Westens « und spielt gleich zweifach der Delegitimierung bestimmter nichtstaatlicher Akteur_innen und der Legitimierung militärischer Gegenmaßnahmen - und damit der Aufrechterhaltung bestehender Herrschaftsverhältnisse - in die Hände (Daase 2011; Winter 2011).

Vor diesem Hintergrund markiert eine postkoloniale Position nicht nur einfach eine Dekonstruktion von Essenzialismen sowie ein Aufbrechen der binären Kodierung des kolonialen Diskurses und »westlicher« Formen der Wissensproduktion. Vielmehr bieten die hier vorgeschlagenen Kritikperspektiven auch eine Verschiebung politikwissenschaftlicher »Sagbarkeitsfelder« (Ziai 2012 a: 293) sowie eine konflikttheoretische Rekonfiguration von Kriegsursachen und Gewaltdynamiken, die sich nicht allein entlang der analytisch gebräuchlichen Achsen lokal/global, staatlich/nichtstaatlich oder politisch/ökonomisch ansiedeln lassen, sondern in Beziehung zur doppelten Gewaltverstricktheit (post-)kolonialer Herrschaftspraktiken und ihrer - von der Wissenschaft teilweise unterstützten oder gar hervorgebrachten - Diskursformationen stehen. Damit greifen wir einerseits auf die bewährte, ältere Erkenntnis der Friedens- und Konfliktforschung zurück, dass konflikttheoretische Analysen die Geschichte des Kolonialismus einschließen müssen, da in dieser historischen Phase die »strukturellen Grundlagen des gegenwärtigen internationalen Systems auf Dauer herausgebildet wurden« und »eine minimale Kenntnis des

3 Im Anschluss an Sebastian Conrad und Shalini Randeria verstehen wir unter Eurozentrismus »die mehr oder weniger explizite Annahme, dass die allgemeine historische Entwicklung, die als charakteristisch für das westliche Europa und das nördliche Amerika betrachtet wird, ein Modell darstellt, an dem die Geschichten und sozialen Formationen aller Gesellschaften gemessen und bewertet werden können« (Conrad/Randeria 2002: 12). 
Kolonialismus unerlässliche Voraussetzung zum Verständnis der Gegenwart ist « (Krippendorff 1986: 102). Andererseits, so unsere These, kann es der Friedens- und Konfliktforschung erst über eine postkolonial informierte Konfliktanalyse gelingen, einen Deutungsrahmen zu entwerfen, der den selbstgeschaffenen Kompetenzproblemen in der Forschung über den Krieg entkommt. ${ }^{4}$

Ausgehend von diesem Verständnis werden wir im Folgenden zunächst jene Diskussionsfelder der Debatte um die »neuen Kriege « in den Blick nehmen, die entlang der Schlagworte »Privatisierung«, »Ökonomisierung« sowie »Brutalisierung« mit eurozentrischen Wahrnehmungsproblemen verwoben sind - und sich in der Konsequenz zu analytischen Deutungsproblemen verdichten. Zwar wurden beide Problemkomplexe von konflikttheoretischer Seite bereits benannt (u. a. Daase 2011; Geis 2006 b; Schlichte 2006), Angebote zu ihrer Überwindung bisher jedoch kaum formuliert. Dies fällt umso schwerer ins Gewicht, da es letztlich um die Kompetenz der Friedens- und Konfliktforschung selbst geht: Inwieweit ist sie einerseits willens, sich der berechtigten Debatte über den Wandel der Kriegsformen analytisch-konstruktiv zu stellen, andererseits aber eben auch fähig, mit einer kritischen Auseinandersetzung und alternativen Deutungsangeboten von Politik und einer breiteren Öffentlichkeit gehört zu werden? Solange die simplifizierende These der »neuen Kriege « im innerwissenschaftlichen Diskurs als ein durchaus geeigneter Interpretationsrahmen erscheint - sei es versatzstückartig als Referenzpunkt zur Erklärung kriegerischer Gewalt in der »Weltgesellschaft « (siehe etwa Bonacker 2006) oder als Baustein in der Konzeption von politikwissenschaftlichen Lehrbüchern (so etwa List 2006) bzw. Nachschlagewerken (Meyers 2011 a) -, sollte es nicht verwundern, dass politische Entscheidungsträger_innen darauf im Prozess der Legitimation militärischer Interventionen zurückgreifen. Die kritische Auseinandersetzung mit den Kernthesen der »neuen Kriege « und ihrer Rezeption in der Friedens- und Konfliktforschung werden wir dann nutzen, um im dritten Kapitel mithilfe postkolonialer Kritikperspektiven sowohl konstruktive Ansatzpunkte für eine Überwindung des Wahrnehmungs- und Kompetenzproblems zu formulieren als auch den konflikttheoretischen Mehrwert postkolonialer Ansätze für die Analyse des (sich wandelnden) globalen Kriegsgeschehens herauszuarbeiten.

4 Dementsprechend ist zu beachten, dass eine kolonialhistorisch informierte Perspektive in den Internationalen Beziehungen, wie Ekkehart Krippendorff sie hier andeutet, keineswegs eine postkoloniale Theorieorientierung miteinschließen muss, was sich beispielsweise im unreflektierten Umgang mit sprachlichen Zuschreibungen zeigt: Krippendorff spricht an anderer Stelle mitunter vom Subkontinent »Schwarzafrika« (1985: 150), von »Eingeborenen« sowie von »primitiven oder wilden Gesellschaften« (1985: 44, siehe insbesondere 1985: Kap. 1) und rekurriert somit selbst auf kolonialistische Diskurs- und Machtformationen. 


\section{Die Debatte um die »neuen Kriege«}

Die Debatte um die »neuen Kriege« verdient heute kaum noch das Prädikat, das sie selbst im Namen trägt. ${ }^{5}$ Angestoßen durch Kaldor (1999) und von Münkler (2002) mit etwas Verzögerung auch in den deutschsprachigen Raum getragen, entwickelten sich in den letzten zehn Jahren weitreichende Diskussionen um den Wandel der Kriegsformen zu Anfang des 21. Jahrhunderts. ${ }^{6}$ Ausgehend von ihren Beobachtungen im Bosnienkrieg zu Beginn und Mitte der 1990er Jahre kam Kaldor zu dem Schluss, dass »neue Kriege « kontrastiert werden können »with earlier wars in terms of their goals, the methods of warfare and how they are financed« (1999: 6). Sie entwickelte zwar keinen kohärenten theoretischen Ansatz, benannte aber klassifizierbare Elemente des gewaltsamen Konfliktaustrags im globalisierten Zeitalter, deren empirische Bezugspunkte sie in der dritten Auflage ihres Werkes auf die komplexen Kriege in Afghanistan und im Irak ausgeweitet hat (Kaldor 2012: Kap. 7). Die Betonung veränderter Kriegsziele (Politik der Identität), Formen der Kriegführung (gezielte Vertreibung) und Elemente der Kriegsfinanzierung (u. a. Diaspora) lassen sich dabei als Versuch begreifen, die vieldeutigen Debatten zur Erklärung der teilweise widersprüchlichen Tendenzen und Entwicklungen im Kriegsgeschehen in den 1990er Jahren zu überwinden und einen Erklärungsansatz anzustreben, der die qualitativen Veränderungen in Akteurskonstellationen und Konfliktdynamiken sowie die neuen lokalen und globalen strukturellen Rahmenbedingungen integriert (Kaldor 2001: 31-33).

Im Unterschied zu Kaldor (1999; 2013) verweist Münkler (2002; 2010;2013) stärker auf die historischen Prozesse der Verstaatlichung und Entstaatlichung politischer Gewalt, die von der einen Kriegsform zur anderen führen. In den heutigen »Staatszerfallskriegen« zeigen sich dann, so Münkler, die gleichen Merkmale der Privatisierung, Ökonomisierung, Sexualisierung und Brutalisierung, die den Krieg in vormoderner Zeit ausgezeichnet hätten (Münkler 2002: 131-173). Bezeichnete Münkler die

5 Für einen Überblick über die Debatte und ihre Kritiker_innen vgl. Benseler et al. (2008); Frech/ Trummer (2005); Geis (2006 a).

6 Dass wir uns in der kritischen Auseinandersetzung primär an Kaldor und Münkler orientieren, liegt eher an der $»$ Verwandtschaft « der Argumentation sowie ihres Einflusses auf wissenschaftliche und politische Debatten, weniger an der Stärke oder Schwäche begrifflich ähnlich gelagerter Deutungsangebote. So nutzen etwa auch Heupel und Zangl (2004) oder Zangl und Zürn (2003) den Typus der »neuen Kriege« zur Sichtbarmachung des »Gestaltwandels kriegerischer Gewalt« - allerdings in einer anderen Variation: Anhand von fünf Kriterien (Kampfverbände, Gewaltmotiv, Gewaltstrategie, Gewaltanwendung und Gewaltökonomie) sollen sowohl die Unterschiede zu »klassischen zwischenstaatlichen Kriegen« als auch zu »klassischen Bürgerkriegen« kenntlich gemacht werden. Den Begriff der »neuen Kriege « nutzen sie dann in einem anderen Sinne: »Neue Kriege« sind Gewaltakte, "um neue Bürgerkriege und den transnationalen Terrorismus zu bekämpfen und um der Spirale von Staatszerfall, Staatsterror und schweren Kriegsverbrechen entgegenzutreten « (Zangl/Zürn 2003: 195). Eine Auseinandersetzung im Kontext einer breiteren typologischen Debatte über den Wandel der Kriegsformen findet sich bei Chojnacki (2007: 487-488). 
»neuen Kriege« dann zunächst noch als »unscharfen, aber offenen Begriff« (2002:9), so spricht er mehr als zehn Jahre später von einer » Theorie der `neuen Kriege« « (2013: 253), welche im Sinne eines Kuhnschen Paradigmenwechsels die etablierten Bestände der Friedens- und Konfliktforschung abzulösen beginne (2013: 260-261). Dass es jedoch angesichts begrifflicher Uneindeutigkeiten, einer Vielzahl an analytischen Widersprüchen sowie einer Reihe an empirischen Unklarheiten wenig Grund für derartige Schlussfolgerungen gibt, werden wir im Folgenden zeigen.

Dazu ist heute mehr denn je ein kritischer Blick auf die Wirkungsmächtigkeit gefragt, die das Narrativ der »neuen Kriege« sowohl in der Wissenschaft als auch in den Medien und im politischen Tagesgeschäft - allen voran bei der Legitimation militärischer Interventionen - entfaltet. ${ }^{7}$ Aus wissenschaftlicher Blickrichtung verbindet sich die These der »neuen Kriege« mit ihrer zentralen Bezugnahme auf die Erosion staatlicher Strukturen in ehemals kolonisierten Räumen erstens mit bereits bestehenden sicherheitspolitischen Diskussionen um "prekäre « oder »fragile« Staatlichkeit (etwa Schneckener 2005; Weiss/Schmierer 2007). Zum anderen werden die »neuen Kriege« als »empirische Trends des globalen Kriegsgeschehens" aufgegriffen, um beispielsweise in theoretischer Absicht Konflikte und Entwicklungen der Weltgesellschaft zu erklären (Bonacker 2006) oder Konzepte einer »Interventionskultur« zu plausibilisieren (Daxner 2010). Drittens finden Begriff und Inhalte der »neuen Kriege « - zumeist gänzlich unhinterfragt - Eingang in Lehr- und Sammelbände sowohl der Internationalen Beziehungen als auch der Friedens- und Konfliktforschung. ${ }^{8}$ Ein Blick auf die anglophone Debatte bestätigt zudem, dass die Arbeiten Kaldors (Dexter 2007, 2008; Reyna 2008) bzw. Münklers (Gregory 2010; Reyna 2009) auch über den deutschsprachigen Raum hinaus rezipiert werden - und dort bereits als neokolonialer Interventionsdiskurs entschlüsselt wurden (insbesondere Dexter 2007; Reyna 2009).

7 Das Friedensgutachten 2013 (Boemcken et al. 2013), das den Titel »Neue Kriege, neue Rüstung, neue Rüstungsmärkte« trägt, steht exemplarisch für eine Vielzahl von Analysen, in denen eine Anknüpfung an die »neuen Kriege« nicht nur auf theoretischer Ebene stattfindet, sondern zugleich zum Anlass genommen wird, Konfliktrealitäten entlang eurozentristisch geprägter Dichotomien - beispielsweise der Trennung zwischen »heroischen« und »postheroischen « Gesellschaften (Boemcken et al. 2013: 6) - zu ordnen und zu analysieren. Offensichtlich wird dabei nahezu ausschließlich der Standpunkt Letzterer (sprich »westlicher « Gesellschaften) eingenommen, wobei die Behauptung der Herausgeber_innen, es gebe einen globalen »Ruf nach einem starken Europa« (Boemcken et al. 2013: 9), ebenso bezweifelt werden darf wie sie vor dem Hintergrund der Ausklammerung jeglicher postkolonialer Betrachtungsweise geradezu zynisch anmutet.

8 Für die Internationalen Beziehungen vgl. Albrecht et al. (2009: 42-44); Krell (2009: 46); List (2006: 69-71) und für die Friedens- und Konfliktforschung vgl. Bonacker/Imbusch (2010: 112); Jahn (2012: 94); Meyers (2011 b: 32-37) sowie Sahm et al. (2006: 9). Gleichermaßen erhellend wie irritierend ist auch ein Blick in das von Dieter Nohlen und Florian Grotz herausgegebene »Kleine Lexikon der Politik«. Hier findet sich nicht nur ein Eintrag zu den »Neuen Kriegen«, sondern dieser stammt auch von Münkler (2011) selbst und lässt nahezu jegliche systematische Kritik aus den Reihen der Friedens- und Konfliktforschung außen vor. 
Jenseits fachwissenschaftlicher Entwicklungen erfreuen sich die Annahmen über einen akteursspezifischen und ökonomisch gelagerten Wandel der Gewaltformen auch im medialen Diskurs ungebrochener Beliebtheit und kreieren damit sowohl einen theoretischen als auch normativ wirkungsmächtigen Debattenhorizont für tagespolitische Streitfragen aus dem Bereich der Sicherheits- und Außenpolitik. Einerseits forderte Münkler dabei selbst die deutsche Bundesregierung zu einer selbstbewussteren Interessenbehauptung auf der internationalen Bühne auf (Münkler 2014 a; Münkler 2014 b). ${ }^{9}$ Andererseits wird das analytische Deutungsangebot der »neuen Kriege« auch von Seiten militärischer Akteur_innen aufgegriffen und unter Bezugnahme auf vornehmlich nationale und regionale Konfliktursachen im »globalen Süden« mit der Notwendigkeit militärischen Gewalthandelns »westlicher« Staaten verknüpft (Gareis 2012). ${ }^{10}$

Angesichts dieser verwobenen fachwissenschaftlichen und politischen Entwicklungen verstehen wir die folgenden Ausführungen keineswegs als reine Rekapitulation bereits vorhandener Kritikperspektiven, sondern als Ausgangspunkt für eine (verbindende) Erweiterung bzw. (Ver-)Schärfung bestehender Kritiken. Hierbei werden wir in einem ersten Schritt (2.1) jene analytischen Perspektiven aufgreifen, die die bisherige Debatte um die »neuen Kriege« prägten: Diese reichen vom unterstellten Wandel auf der Akteursebene bzw. im Vergesellschaftungsgrad und -verhältnis der beteiligten Gewaltgruppierungen (»Privatisierung«), über die damit behaupteten Transformationsprozesse im Verhältnis von Krieg, Politik und Ökonomie und die daran angebundenen Handlungslogiken nichtstaatlicher Akteur_innen (»Ökonomisierung «) bis hin zu den unterstellten Veränderungen der Gewaltdynamiken im Krieg (»Brutalisierung «). ${ }^{11}$ Dazu werden wir auch die bereits bestehenden Einwände der Friedens- und Konfliktforschung kritisch einordnen. Es

9 Die mediale Präsenz der »neuen Kriege« wird zudem durch eine Reihe von Publikationen der Bundeszentrale für politische Bildung verdeutlicht (Bundeszentrale für politische Bildung $2007 \mathrm{a}$; 2007 b; 2011 a; 2011 b), die lange Zeit auch Münklers Monographie »Die neuen Kriege« vertrieb (Ziai 2012 b: 296).

10 Eine gewinnbringende Analysestrategie, der narrativen Wirkmächtigkeit der »neuen Kriege« weiter nachzugehen, könnte somit in einem von Bialasiewicz et al. (2007) vorgeschlagenen Ansatz liegen, der die performativen und diskursiven Resonanzwirkungen zwischen Wissenschaft, Politik und Medien als wirkungsvollen Mechanismus zur Verfestigung und Materialisierung vielfältig gelagerter Macht- und Herrschaftsverhältnisse begreift.

11 Auf die Auseinandersetzung mit anderen zentralen Thesen (wie der Kennzeichnung »neuer Kriege« durch »Asymmetrie«) sowie deren Verbindung zum transnationalen Terrorismus (Münkler 2002: Kap. 5) verzichten wir hier weitgehend. Einerseits sind diese Aspekte schon an anderer Stelle einer ausführlichen kritischen Bestandsaufnahme unterzogen worden (u. a. Chojnacki 2004; Kahl/ Teusch 2004). Andererseits ergeben sich diese Argumente teilweise direkt aus den Annahmen der »Privatisierung« und »Ökonomisierung«, sodass wir in diesem Beitrag dort darauf Bezug nehmen, wo uns eine kritische Stellungnahme relevant erscheint. 
geht uns dabei jedoch nicht allein um eine Reproduktion jener Argumente, die mit den veränderten medialen und wissenschaftlichen Blickwinkeln auf kriegerische Gewalt nach dem Ende der Blockkonfrontation von 1989/90 zusammenhängen und auf Verkürzungen und Stereotype innerhalb der »westlichen« Berichterstattung über Konflikträume der »Anderen« zurückzuführen sind (u. a. Kahl/Teusch 2004; Kalyvas 2001; Newman 2004). Vielmehr wollen wir auch dechiffrieren, wie die verwendeten Begriffe und Perspektiven mit eurozentrisch verengenden und machtpolitisch wirkungsmächtigen Diskursformationen verstrickt sind. Bevor wir dann im dritten Kapitel die hier in den Mittelpunkt gestellten Analysedimensionen »Privatisierung «, "Ökonomisierung « und »Brutalisierung « aus postkolonialen Theorieperspektiven kritisch hinterfragen, werden wir in einem Zwischenschritt (2.4) noch einmal zeigen, wie sich die Wahrnehmungs- und Deutungsprobleme auf theoretischer Ebene fortsetzen bzw. sogar verschärfen.

\subsection{Privatisierung}

Im Kern beruht die These der »neuen Kriege « auf dem akteurszentrierten Argument der quantitativen Zunahme und Diversifizierung nichtstaatlicher Gewaltgruppierungen (Münkler 2008: 27-32). Nicht Staaten, vielmehr Warlords, terroristische Netzwerke, kommerzielle Sicherheitsunternehmen und substaatliche Gewaltgruppierungen sowie Kindersoldat_innen bestimmten das Kriegsgeschehen (Münkler 2002: 33-43; 2010: Kap. 15). Die Zivilisierung und Professionalisierung des kämpfenden Soldaten sowie die Institutionalisierung des Militärs, die charakteristisch für die Verstaatlichung des Krieges waren, würden in den »neuen Kriegen « zunehmend aufgelöst und die mit diesen Eigenschaften verbundenen Begrenzungen des Krieges wie die Unterscheidung zwischen Zivilist_innen und Kombattant_innen verlieren, so Münkler (2002: 108-118; 2010: 313), ihren diskriminierenden Charakter. Die unterstellten Prozesse von Entstaatlichung und Privatisierung kriegerischer Gewalt führen dann zur Schlussfolgerung, dass Staaten - im Unterschied zur Ära der Staatenbildungskriege, die empirisch das Kriegsgeschehen bis zur Phase der Dekolonisierung bestimmt hätten - nicht mehr die selbstverständlichen Monopolisten der Kriege seien (Münkler 2002: 18-22). Die Deutung der meisten Kriege der Gegenwart als Staatszerfallskriege steht dabei ganz offensichtlich in unmittelbarer Verwandtschaft zum Konzept der failed states, das im internationalen Vergleich eine ähnliche Karriere hinter sich hat (Goldstone 2008; Rotberg 2007), aber nicht weniger problematisch ist (siehe unten).

In dem Maße, in dem der »alte « Staatenkrieg zum »historischen Auslaufmodell« (Münkler 2002: 7) avanciert, wird gleichzeitig unterstellt, dass die staatszentrierten 
und staatserhaltenden innerstaatlichen Kriege entweder an Relevanz verlören oder im Kontext der »neuen Kriege « zu deuten seien. Diese Argumentation täuscht freilich darüber hinweg, dass empirische Studien zu weit weniger eindeutigen Ergebnissen kommen: Zwar treten zwischenstaatliche Kriege seit Ende des Kalten Krieges tatsächlich deutlich seltener auf als innerstaatliche; das Uppsala Conflict Data Program zählt für das Jahr 201232 bewaffnete Konflikte, von denen 31 innerhalb staatlicher Grenzen ausgetragen wurden (Themnér/Wallensteen 2013). Feststellungen dieser Art reichen jedoch nicht aus, um den Entwicklungen gerecht zu werden, zumal innerstaatliche Gewaltformen auch schon lange vor Ende des Kalten Krieges fester Bestandteil des globalen Kriegsgeschehens waren und seit mehr als zwei Jahrhunderten das Kriegsgeschehen nachhaltig prägen (Chojnacki 2006: 49; Kahl/Teusch 2004: 388; Rasler/Thompson 2011; Sarkees 2014). Weitaus problematischer ist, dass die »neuen Kriege« mit ihrer These einer Erosion von Staatlichkeit in Form sogenannter failed states in ein verkürztes Kriegsverständnis steuern, das Kriege vornehmlich entlang ihrer vermeintlichen Entstaatlichung erfasst, die staatserhaltende und staatsbildende Prozesse der übergroßen Mehrzahl innerstaatlicher Kriege unterschlägt (Sarkees 2014:244) sowie die unterschiedlichen Formen und Funktionen von Gewalt im Krieg ausblendet (u. a. Kalyvas 2006; Tilly 2003).

Konfliktanalytisch differenziertere Beiträge haben eine solche Einengung auf das Phänomen failed states zu Recht als Wahrnehmungsproblem der »westlichen « Wissenschaftswelt entschlüsselt (Daase 2011; Geis 2006 b; Schlichte 2006). Im Mittelpunkt der Kritik steht hier das Argument, dass mit dem einseitigen Fokus auf staatliche Zerfallsprozesse im »globalen Süden« Kriege außerhalb der OECD-Welt in generalisierender und simplifizierender Weise mit den destruktiven Effekten »staatlichen Versagens« in Verbindung gebracht werden. Eine solche Verknüpfung läuft nicht nur Gefahr, monokausale Vereinfachungen als Erklärung des Kriegsgeschehens anzubieten, sondern reproduziert auch schlicht die Wirkmächtigkeit einer »eurozentrischen Idealisierung von Staatlichkeit« (Daase 2011: 23) - mit der Konsequenz, dass Friedens- und Ordnungsvorstellungen jenseits europäischer Modellvorgaben aus dem Wahrnehmungsraster »westlicher « Analysestrategien fallen (Geis 2006 b: 14-17). Gleichzeitig werden bestimmte »neue« Konfliktkonstellationen durch die Verengung auf »Krieg als Geschäft « ebenso depolitisiert wie die darin eingewobenen nichtstaatlichen Gewaltakteur_innen delegitimiert werden (Winter 2011). Weite Teile der Friedens- und Konfliktforschung müssen sich daher selbst fragen, warum ihnen offensichtlich erst nach dem Ende des Ost-West-Konflikts klar geworden ist, »dass die Welt gar nicht in dem Maße verstaatlicht ist, wie die gängigen Weltkarten und Redeweisen dies nahe legen « (Schlichte 2006: 116). So wie das Argument der Privatisierung nur vor dem Hintergrund von Verstaatlichung 
funktioniert und Sinn ergibt, kann auch nur zerfallen, was vorher schon als (nicht nur de jure) politische Organisationsform da war (Gantzel 2002: 10). Aus diesem Grund betonen alternative Sichtweisen die Möglichkeit von Staatsbildungskriegen oder von unvollendeter Staatlichkeit in den Staaten des »globalen Südens « (Schlichte 2006: 115). Zusätzlich zeigt Meredith Sarkees (2014) auf Basis der Correlatesof-War-Daten aus empirisch-systematischer Perspektive, dass die mit Abstand meisten innerstaatlichen Kriege seit 1816 (und auch in der Phase seit dem Ende des Zweiten Weltkriegs) auf die Kriegstypen »Kämpfe um zentrale Kontrolle« und »lokaler/regionaler Systemwandel oder Sezession « entfallen. Errol Henderson und J. David Singer (2002: 186) unterstreichen ebenfalls aus empirisch-systematischer Perspektive, dass die Korrelate und Ursachen der angeführten »neuen Kriege« jenen Gewaltkonflikten, die entlang etablierter, an Staatlichkeit orientierter Typologien eingeordnet werden (zwischenstaatlich, innerstaatlich, extrastaatlich), sehr ähnlich sind. Im Ergebnis entpuppen sich die »neuen Kriege« dann als nichts anderes als ein »amalgam of different types of >old wars «" (Henderson/Singer 2002: 166) jedoch mit dem entscheidenden Nachteil, dass die Differenzierungen zwischen den genannten Kriegstypen in der grobkörnigen Gegenüberstellung »alter « und »neuer Kriege « verloren gehen - und damit nicht zuletzt die historische Realität kolonialer Befreiungskriege (extrastaatlicher Krieg) unterschlagen zu werden droht. ${ }^{12}$

Welche vorläufigen Schlussfolgerungen lassen sich aus diesen Überlegungen ziehen? Zum einen wird die vereinfachende Typologie »neue vs. alte Kriege« den Komplexitäten im empirischen Kriegsgeschehen, insbesondere im Verhältnis von Staat und Krieg, nicht gerecht. Gleichzeitig werden Ähnlichkeiten zu den Akteurs-, Struktur- und Prozessfaktoren »alter Kriege" verdeckt und weiterführende komparative Analysen durch die Amalgamierung vielförmiger Gewaltkonstellationen zu »neuen Kriegen« erschwert oder gar verhindert. Zum anderen täuscht eine solche binäre Kodierung darüber hinweg, dass Prozesse der Ver- bzw. Entstaatlichung postkolonialer Räume eine Geschichte haben, die über die Zäsur des Kalten Krieges hinausweist. Dies bedeutet dann keinen Rückfall in die Analyseebenen und Kriegstypologien der »Staatenwelt« mit ihrer Leitkategorie »staatlich vs. nichtstaatlich «, sondern verdeutlicht vielmehr die Notwendigkeit ihrer analytischen Einbettung in die historischen (Dis-)Kontinuitäten postkolonialer Gewaltverhältnisse und »westlicher « Wissensproduktion. Damit sind Konzepte gefordert, die die historisch spezifische und mit materiellen Machtverhältnissen verbundene Entwicklung

12 Dass die empirische Kriegsursachenforschung hier ein erheblich feineres Typologie-Instrumentarium entwickeln konnte, das von intra-state wars und extra-state wars über sub-state wars bis zu den unter der Kriegsschwelle angesiedelten Militarized Interstate Disputes (MIDs) reicht, scheint Münkler nur wenig zu interessieren (Gärtner 2008: 64). 
(post-)kolonialer Staatlichkeit zum Ausgangspunkt (und nicht zum Problemfall) ihrer Überlegungen machen.

\section{2 Ökonomisierung}

Im Verbund mit dem Argument der Privatisierung wird den »neuen Kriegen« eine zunehmende Ökonomisierung von Handlungslogiken und Rahmenbedingungen unterstellt. Illegaler Rohstoffhandel, Schmuggel, Drogengeschäfte und die Anknüpfung an die global organisierte Kriminalität tragen, so die These, zu ökonomisch motiviertem Konflikthandeln sowie zu einer Transnationalisierung vielfältig vernetzter Kriegswirtschaften bei (Münkler 2013: 253-254; 2002: Kap. 4). Dass die »neuen Kriege« dabei weniger im politischen Kontext ehemaliger Partisanenkriege, sondern vielmehr in Zusammenhang mit wirtschaftlichen Interessenlagen betrachtet werden müssen, liegt Münkler zufolge in der neuen Rentabilität der Kriege, die auf die gesunkenen Kosten für militärische Einsatzmittel zurückzuführen sei: Leichte, auf illegalem Wege beschaffte Waffen und die vergleichsweise kostengünstige Rekrutierung sozial entwurzelter männlicher Soldaten trügen ihren Teil zur Ökonomisierung des Krieges bei (Münkler 2002: 136-138). Gleichzeitig hätten die Veruntreuung »westlicher « Hilfslieferungen und die Abwesenheit übergeordneter staatlicher Herrschaftsstrukturen ihren Anteil daran, dass sich der Krieg in $»$ Krisengebieten « zu einer dauerhaften Form des Wirtschaftens verfestige (Münkler 2002: 153-159). Was dabei entstehe, seien offene Kriegsökonomien, in denen Kriege nicht länger der Durchsetzung politischer Interessenlagen dienten, sondern als global vernetzte Finanzierungspraktik privater Kriegsunternehmer_innen verstanden werden müssten (Münkler 2002: 159-173; 2010: 291-298).

Gegen diese verengte Sicht auf ökonomische Gewaltmotive wandten Kritiker_innen ein, dass die einseitige Fokussierung auf Motive der »Gier« einzelner Konfliktakteur_innen der komplexen Struktur verschiedenartiger und sich gegenseitig bedingender Handlungsmotivationen nicht gerecht werde (etwa Kahl/Teusch 2004: 394-400; Matthies 2004: 186-187; Veit 2008). ${ }^{13}$ Anstatt politische, ökonomische und anders gelagerte Motivlagen in ihrer wechselseitigen Bedingtheit zu analysieren, droht die Debatte hier zudem in eine dichotome Gegenüberstellung von Politik auf der einen und Ökonomie auf der anderen Seite zu steuern (Daase 2011:24). Dies

13 Münkler kritisiert selbst die Einseitigkeit, mit der nach Ende des Kalten Krieges sogenannte »ethnische « und religiös-kulturelle Erklärungsmuster herangezogen wurden, um die Leerstelle zu füllen, welche sich durch den Wegfall der beiden politischen Großideologien ergab (2002: 159-161). So richtig Münkler damit liegt, auf die Einseitigkeit eines solchen Vorgehens hinzuweisen, so wenig hilfreich ist es jedoch, die Einengung auf »ethnisch-religiöse« Erklärungsmuster wiederum durch einen ökonomischen Monismus zu ersetzen (Schlichte 2006: 117). 
führt erstens dazu, dass mit der Zuschreibung ökonomischer Handlungsmotivationen zugleich eine politische Delegitimierung der als »gierig « markierten nichtstaatlichen Gewaltakteur_innen einhergeht, »denen der Wille und die Fähigkeit zur MäBigung abgesprochen werden « (Daase 2011: 24). Zweitens verkennt ein wie auch immer gearteter ökonomischer Determinismus die organisationsspezifischen Logiken und ideologischen Quellen gewaltförmigen Handelns (Malešević/Dochartaigh 2014: 61). Empirische Konfliktstudien haben zahlreiche Nachweise erbracht, dass gerade auch die Organisationsform der Gewaltgruppierungen und die Art der Rekrutierung jenseits ökonomischer Motive den Charakter des Gewalthandelns maßgeblich beeinflussen (u. a. Azam 2006; Weinstein 2007).

Der Eindruck, dass private und staatliche Motivlagen durchaus unterschiedlichen Bewertungsmodi unterliegen, verstärkt sich zusätzlich, wenn die Perspektive verlagert und danach gefragt wird, wie die materiellen Interessen staatlicher Gewaltinstitutionen und »westlicher« Rüstungs- und Sicherheitsmärkte in der Forschung zu den »neuen Kriegen « verhandelt werden: meist unkritisch, verkürzend oder gar irreführend (Schlichte 2006: 117-119). Werden beispielsweise kommerzielle Sicherheitsfirmen, die im staatlichen Auftrag des »globalen Nordens« militärische Dienstleistungen anbieten, als Argument für die Zunahme ökonomischer Motive herangezogen, dann werden sie zum einen in eine aus historisch-vergleichender Perspektive problematische und eher romantisierende Linie mit den Condottieri des späten Mittelalters und der frühen Neuzeit gestellt (Münkler 2013: 256). Wobei jedoch leicht übersehen wird, dass die Condottieri im Unterschied zu den heutigen Sicherheitsfirmen ihre militärischen Fähigkeiten durch vielfältige persönliche, politische und ökonomische Netzwerke durchaus auch in politische Herrschaft zu transformieren vermochten und damit als $\gg$ Katalysatoren staatsgenetischer Prozesse« gedeutet werden können (Lang, zitiert nach Pöhlmann 2006). Zum anderen wird der Einsatz privater Sicherheitsfirmen als »feine und saubere Form « des Kriegsgeschehens sprachlich bedenklich und analytisch sinnfrei der $»$ schmutzigen Form der Kommerzialisierung« der Warlords gegenübergestellt (Münkler 2013: 256).

Die These der Ökonomisierung der »neuen Kriege« markiert daher ein doppeltes konflikttheoretisches Problem: Erstens übersieht die Fokussierung auf finanzielle Handlungslogiken des Krieges nichtökonomische Motivlagen sowie die alternativen Herrschaftspraktiken nichtstaatlicher Gewaltakteur_innen jenseits oder unterhalb des Staates. Zweitens droht so eine latente Entpolitisierung und Delegitimierung solcher Gewaltgruppierungen, die nicht mit den gängigen Ordnungsvorstellungen »westlicher « Politik und Wissenschaft übereinstimmen. ${ }^{14}$ Dass auch heutige

14 Siehe hierzu Abschnitt 3.2. 
Kriege nach wie vor politischen Handlungsmotivationen unterliegen, steht selbst für konkurrierende Ansätze der vergleichenden Kriegsforschung außer Frage (u. a. Kalyvas 2006; Schlichte 2006; Weinstein 2007; Wood 2008). Nicht sicher ist jedoch, ob die »westliche « Wissenschaftswelt dazu in der Lage ist, diese auch zu erkennen: »Die Motive der Kriegsakteure sind auch heute noch politisch, aber ihre Politizität fügt sich nicht immer westlichen Schemata« (Schlichte 2006: 117). ${ }^{15}$

\subsection{Brutalisierung}

Eine dritte Argumentationslinie der Rede von den »neuen Kriegen« zielt schließlich auf eine veränderte Wahrnehmung und Deutung der Dynamiken im Krieg, insbesondere auf eine Brutalisierung und Asymmetrierung der Kampfhandlungen. Die Brutalisierung der »neuen Kriege«, welche sich nicht auf Kombattant_innen beschränken ließe, sondern gerade in der Gewalt gegen die Zivilbevölkerung eine neue Qualität erreiche, wird von Befürworter_innen der »neuen Kriege« wiederholt betont (etwa Heupel/Zangl 2004; Münkler 2013; 2002: 142-153; kritisch: Gantzel 2002; Kahl/Teusch 2004). Besondere Aufmerksamkeit schenkt Münkler hierbei der »Sexualisierung" von Gewalt, die in »fast allen neuen Kriegen zu beobachten" (2002: 151) sei und in Widerspruch stehe zu den »klassischen « Staatenkriegen Europas, in denen sexualisierte Gewalt aufgrund militärisch-taktischer Erwägungen sowie völkerrechtlicher Einschränkungen nie die gleichen Ausmaße erreicht habe (2002: 148; 2010: Kap. 15). Gemäß der Annahme einer Entgrenzung der Gewalt trete sexualisierte Gewalt in den »neuen Kriegen« hingegen als selbstzweckhafte »Form des Schmerzzufügens und Quälens« (2002: 151) auf, bei der nicht weiter die militärische Ausschaltung der Gegner_innen im Vordergrund stehe, sondern Lustempfinden, Macht und Gewalt - für Münkler eine »Rückkehr zu den Formen spätmittelalterlicher Kriegführung « (2002: 148). Begleitet wird die These der Brutalisierung durch problematische Pauschalisierungen, wenn etwa die »neuen Kriege« verallgemeinernd als »Massakerkriege « (Münkler 2013: 253) gekennzeichnet oder auf der Folie eines binären Framings zivilisierter vs. nichtzivilisierter Formen der Kriegführung interpretiert werden. So werden die »neuen« Konfliktkonstellationen

15 Solange implizit oder explizit das Modell des europäischen Nationalstaats als analytische Folie zugrunde gelegt und mit einem Politikverständnis verbunden wird, das Politik über die Gewährleistung administrativer, am Gemeinwohl orientierter Entscheidungen definiert, bleibt meist unklar, was eigentlich wie in den betroffenen sozialen Räumen durch politische Praktiken realisiert wird. Auswege aus dieser Sackgasse bzw. alternative Blickwinkel bieten etwa Mielke et al. (2011) mit dem Konzept der durch sozio-politische Entitäten strukturierten social orders oder William Reno (2011) mit einer Analyse der vielfältigen, gewaltförmigen Politikpraktiken im Anschluss an die Unabhängigkeit vieler politischer Einheiten auf dem afrikanischen Kontinent. 
in den gescheiterten Räumen der »Anderen«, die von »bestialische[n] Gewalthandlungen « [Anm. d. A.], von »Mord und Verstümmelung, Folter und Vergewaltigung « (Heupel/Zangl 2004: 354-355) gekennzeichnet seien, in den moralisch vermeintlich überlegenen Rahmen der »westlichen« Moderne eingepasst (Daase 2011: 22; Winter 2011: 490).

Dass das Bild »kriegshungriger Kämpfer« jedoch nicht nur rhetorisch problematisch, sondern auch analytisch irreführend ist, wird beim vorschnellen sprachlichen $\mathrm{Zu}$ - und Festschreiben von essenzialisierten Identitäten und scheinbar irrationalen Handlungsmustern deutlich. Dabei sind der Rückbezug auf Identitäten (Kaldor 1999) oder die unterstellte »Brutalisierung« (Münkler 2002) für sich genommen noch keine Indizien für die Irrationalität der handelnden Individuen und Gruppen in gewaltsamen Konflikten. Wird von der Annahme ausgegangen, dass organisierte Gewaltakteur_innen auf ein breites Repertoire von Gewaltstrategien zurückgreifen können (Tilly 2003) und dass in innerstaatlichen Kriegen staatliche wie nichtstaatliche Akteur_innen intendiert Gewalt gegen konkurrierende und eigene Gruppen zur Kontrolle von territorialer Gebietshoheit anwenden (Kalyvas 2006), dann wird der analytische Blick frei für den Organisationsgrad politischer Einheiten, die Präferenzen politischer Eliten sowie für ihre gewaltförmigen Handlungsstrategien gegen militärische und zivile Ziele. Soziologische wie politikwissenschaftliche Studien belegen, dass Gewalt - selbst in den heftigsten Ausformungen gegen die Zivilbevölkerung - durchaus identifizierbaren Handlungskalkülen folgt und häufig der Demonstration von Macht sowie der Rekrutierung neuer Kämpfer_innen dient (u. a. Azam 2006; Elwert 1997) oder aber eine Reaktion auf zuvor verlorene Kämpfe über territoriale Kontrolle ist (Eck/Hultman 2007; Kalyvas 2006). Zudem muss berücksichtigt werden, dass der Einsatz gezielter Gewalt gegen Zivilist_innen ein wiederholtes Muster aller Kriegstypen über Raum und Zeit ist (Newman 2004: 181). Wer also Konfliktkonstellationen und Gewaltstrategien in ein problematisches Schema »normativer Asymmetrie« moralisch einbettet (Daase 2011: 22), enthebt sich selbst der Möglichkeit, die - hier nur angedeuteten - vielfältigen Dynamiken der Kriegführung zu verstehen und beobachtbare raum-zeitliche Varianzen konflikttheoretisch zu erklären. Dies gilt im Übrigen auch für die These der »Resexualisierung der Gewalt« (Münkler 2002: 142-153) und das damit einhergehende simplifizierende Verständnis von Geschlecht und Krieg. Gerade Männlichkeitskonstruktionen, die an aggressive Aktivität sowie Kämpferrollen geknüpft sind, fungieren in vielen Konfliktkonstellationen als ein subtiler Rekrutierungsmechanismus sowohl für »reguläre « Armeen als auch für nichtstaatliche Konfliktparteien (etwa Blagojević 2004). Wie Studien darüber hinaus belegen, ist sexualisierte Gewalt gegen Frauen (aber auch Männer) in vielen Kriegen zum einen ein strategisches 
Instrument, um Macht und Kontrolle sowohl nach innen als auch nach außen gegenüber als schwach und verletzlich markierten Gruppen zu demonstrieren (Zarkov 2001). Zum anderen sind Vergewaltigungen in Kriegszeiten Teil der diskursiven Konstruktion kollektiver Identität und ein einflussreiches politisches Mittel der Konstruktion von Feindbildern (Baines 2003; Oberschall 2000: 991). Diese Prozesse sind jedoch alles andere als neu und variieren, wie die empirische Forschung zeigt, von Fall zu Fall (Seifert 2002; Wood 2006).

Zur konzeptionell wie empirisch wenig überzeugenden These zunehmend brutalisierter Kriege gesellt sich noch die problematische Vergleichsfolie der »alten Kriege« bzw. die Verhältnismäßigkeit der Darstellung kriegerischer Gewalt innerhalb eigener kultureller und historischer Erfahrungshorizonte. Offensichtlich kann doch nur so lange von einer gesteigerten Brutalität der »neuen Kriege« gesprochen werden, wie gleichzeitig einer geschönten europäischen Kriegsvergangenheit das Wort geredet wird (Geis 2006 b: 15; Plumelle-Uribe 2004). Die Idee einer gehegten europäischen Kriegsgeschichte täuscht jedoch nicht nur über die historischen Realitäten des Ersten und Zweiten Weltkrieges sowie über die (post-)koloniale Vergangenheit und Gegenwart Europas und Deutschlands hinweg, ${ }^{16}$ sondern rückt wie am Beispiel Münklers (2013) aufgezeigt werden kann - Konfliktanalysen auch in gefährliche Nähe zur Legitimation zukünftiger Militärinterventionen. Jenseits der »historischen Amnesie« der These von den »neuen Kriegen« (Kennedy/Waldman 2014: 220) lassen sich die »Brutalisierung des Krieges « und die »Pazifizierung unbotmäßiger Völker« entsprechend als strukturelle Probleme im modernen internationalen System identifizieren (Krippendorff 1977: 105), deren Folgen nicht zuletzt durch den Mythos eines "gehegten « Staatenkrieges verdeckt worden sind. ${ }^{17}$ Wird darüber hinaus berücksichtigt, dass Bilder terroristischer Gewalt in Zeiten massenmedialer Vernetzung eine enorme Wirkungskraft erlangt haben und die diskursiven Umrahmungen kriegswissenschaftlicher Realitäten somit zunehmend in den Mittelpunkt kriegslegitimierender Praktiken rücken (etwa Bergmann 2010; Thiele 2010), wird es möglich, die Schreckenserzählung des transnationalen Terrorismus

16 Wie Rosa A. Plumelle-Uribe (2004) zeigt, erfahren europäische Kolonial- und Gewaltverbrechen bis heute keine angemessene Aufarbeitung, sondern werden mit dem Verweis auf heroische europäische »Entdecker« romantisch verklärt. Ebenso wird gerade deutsche Kolonialgeschichte bis heute weitgehend marginalisiert und unter dem Deckmantel der Untaten anderer Kolonialmächte gehalten (vgl. hierzu Ofuatey-Alazard 2011 sowie Sow 2011).

17 Dass die räumlich, zeitlich wie sektoral (politisch, ökonomisch) ungleichförmigen Entwicklungen der Staatenbildung im 19. und frühen 20. Jahrhundert sowie die damit einhergehenden Expansionen und Kolonialkriege der Großmächte zu vielfältigen »Typen der Gewalt in staatsfernen Räumen« geführt haben, zeigt beispielsweise Jörg Baberowski (2007) anhand der Kriege Russlands und der Sowjetunion (1905-1950), die jeweils auch Kriege gegen Zivilist_innen waren. 
als politisch gewollte »Erzählung der Angst« (Schlichte 2006: 127) zu erkennen. Klaus Schlichte betont daher zu Recht die Notwendigkeit, die Wirkungszusammenhänge eines Narrativs zu untersuchen, das transnationalen Terrorismus, fragile Staatlichkeit und die »neuen Kriege« zu einem »sicherheitspolitischen Gesamtkomplex « (2006: 126) verschmilzt und damit zunehmend die Form eines Legitimationsdiskurses »westlicher« Militärinterventionen annimmt. Wir werden diesem begründeten Verdacht an späterer Stelle (3.3) genauer nachgehen, wenn wir mithilfe postkolonialer Theorieansätze die Parallelitäten zwischen den diskursiven Gewaltund Legitimierungsstrategien europäischer Kolonialbestrebungen auf der einen und der »neuen Kriege« auf der anderen Seite genauer sichtbar machen.

\subsection{Theoretische Ausgangspunkte der »neuen Kriege»}

So augenfällig die methodischen und analytischen Probleme entlang der oben skizzierten Diskussionsfelder sind, so weitreichend sind auch die theoretischen Implikationen - oder anders formuliert: so offenkundig wird auch, dass die klassifikatorische Unterscheidung in »alte « und »neue Kriege« chronisch untertheoretisiert ist. An diesem Befund ändert auch der jüngst formulierte Anspruch Münklers wenig, mit den identifizierten Dimensionen des Wandels des Kriegsgeschehens der Friedens- und Konfliktforschung eine »Theorie der sneuen Kriege« « und damit gar ein »neues Forschungsparadigma« geliefert zu haben (2013: 261-262).

Die eindeutige Identifikation und dichte Beschreibung mehrdimensionaler Merkmalsräume (wie z. B. von nichtstaatlichen Akteurskonstellationen oder Prozessen der kriegsökonomischen Globalisierung) sind zweifellos wichtige Schritte zur Bildung von Typologien. Ebenso können Typologien ein wichtiges Bindeglied zwischen der Ordnung empirischer Beobachtungen und der Bildung neuer Theorien sein und zur theoretisch geleiteten Überprüfung wichtiger Hypothesen anregen. Die Verdichtung bestimmter empirischer Merkmale zu einer deskriptiven Typologie rechtfertigt aber weder eine »neue Theorie« noch ein »neues Forschungsparadigma«. Stattdessen müsste das behauptete Zusammentreffen einzelner Charakteristika für theoriebildende Zwecke nicht nur in einen systematischen Zusammenhang von Ursache-Wirkungsbeziehungen gesetzt werden, sondern die daraus ableitbaren Hypothesen müssten auch empirischen Überprüfungsschritten standhalten. Beide Aspekte bleiben Kaldor und Münkler schuldig. Damit verbunden ist eine doppelte Leerstelle: Einerseits erfolgt keine systematische Rückkopplung der diskutierten analytischen Charakteristika an andere, teilweise konkurrierende theoretische Erklärungsansätze oder Hypothesen zur Entwicklung des globalen Kriegsgeschehens innerhalb des disziplinären Subfeldes der vergleichenden Kriegsforschung; 
andererseits mangelt es an einer konkreten Ausformulierung dessen, wie die identifizierten analytischen Dimensionen »neuer Kriege« nicht nur additiv und beschreibend zur Prägung eines bestimmten Typus von Krieg genutzt, sondern auch in ein kohärentes wissenschaftliches Aussagesystem überführt werden können.

Auch der fehlende Einbezug von Erkenntnissen anderer - vor allem auch internationaler - Forschungsströmungen mag auf den ersten Blick überraschen, ist aber für die Friedens- und Konfliktforschung alles andere als neu (siehe zum Beispiel die Ignoranz der Arbeitsgemeinschaft Kriegsursachenforschung (AKUF) gegenüber den Forschungsergebnissen des Correlates-of-War-Projektes). So falsch es dabei ist, pauschal zu unterstellen, dass sich »Teile der etablierten Friedens- und Konfliktforschung durch die Theorie der >neuen Kriege in ihrer Existenzberechtigung bedroht sahen « (Münkler 2013: 259), so offenkundig vorschnell und falsch ist die Schlussfolgerung, dass die Erklärungsansätze zum Demokratischen Frieden durch das bloße Beobachten eines gewandelten, neuen Kriegsbildes »obsolet« seien (Münkler 2013: 260). ${ }^{18}$ Redlicher wäre es zunächst einmal, sich an jenen Erkenntnissen abzuarbeiten und zu reiben, die sich mit dem Nexus bestehend aus dem Wirken diverser nichtstaatlicher Kriegsakteur_innen, den Ursachen und Folgen von Kriegsökonomien sowie den damit verbundenen Gewaltstrategien beschäftigen. Dabei würde schnell deutlich, dass die in der vergleichenden Kriegsforschung ermittelten empirischen Varianzen auf den Ebenen der Konfliktakteur_innen und ihrer Motive (u. a. Blattman/Miguel 2010; Buhaug 2010; Garfinkel/Skaperdas 2006; Weinstein 2007) sowie der strukturellen Charakteristika von Kriegsökonomien (u. a. Koubi et al. 2013; Le Billon 2007; Ross 2004), aber auch die relevanten Konfliktdynamiken unter Berücksichtigung von Drittparteien (intervenierende Großmächte, ehemalige Kolonialmächte, Nachbarstaaten oder internationale Organisationen) den Schritt von einer grobkörnigen deskriptiven Typologie (»alt vs. neu«) hin zur Theoriebildung nicht zulassen.

Aber was wäre dann überhaupt mit einer Theorie »neuer Kriege« gewonnen, die die wesentlichen Unterschiede in den Ursachen und Ausprägungen (Intensitäten und Dauer) gegenwärtiger Kriege nicht zu erfassen vermag bzw. diese nicht einmal problematisiert? Diese Frage kann noch weiter zugespitzt werden, wenn berücksichtigt wird, dass die »Charakteristika« der »neuen Kriege« auf unterschiedlichen

18 Münkler übersieht (oder verschweigt) hier zudem, dass es sich bei den Erklärungen zum Demokratischen Frieden nicht um ein kohärentes Theoriegebäude handelt, sondern eher um sich teils ergänzende, teils konkurrierende Ansätze. Herausgefordert im Sinne der Theoriebildung werden diese Ansätze auch weniger von Überlegungen zu »neuen Kriegen«, sondern vielmehr von der Einsicht, dass eine irgendwie geartete Theorie des Demokratischen Friedens auch die Demokratischen Kriege erklären müsste (Daase 2006). 
Ebenen und teilweise auch Ontologien angesiedelt werden: So wird den beteiligten Akteur_innen (gemeint sind aus androzentristischer Perspektive meist männliche Kämpfer) im Zusammenhang mit der Herausbildung von Kriegsökonomien einerseits rationales und ökonomisch motiviertes Gewalthandeln zugesprochen (Münkler 2013: 259), andererseits wird es ihnen dort wieder abgesprochen, wo die Thesen der Barbarisierung und Resexualisierung von Gewaltpraktiken durchscheinen und wo keine definierten »Ziele und Zwecke auszumachen « sind, um »derentwillen der Krieg geführt wird« (Münkler 2002: 31). Ein ähnlich gelagertes Problem entsteht bei der Argumentationsfigur der »Kommerzialisierung«, die unterschiedliche Akteursformationen und -logiken zu vereinen sucht, indem sie die Auslagerung militärischer und sicherheitsrelevanter Aufgaben an zunehmend diversifizierte Sicherheits- und Militärfirmen in einen systematischen Zusammenhang zum »Eindringen wirtschaftlicher Motive in die unmittelbare Kriegsführung « (Münkler 2013: 256) stellt. Dies geschieht jedoch zu Lasten theoretischer Genauigkeit, da hier zu differenzierende Dynamiken vermischt werden: Auf der einen Seite steht das intendierte Handeln lokaler Gewaltakteur_innen, die globalisierte Kriegsökonomien für ihre eigenen Interessen und ihr eigenes Überleben nutzen - in den Worten des »westlichen " Analytikers: die »schmutzige" Form der Kommerzialisierung (Münkler 2013: 256). Auf der anderen Seite stehen die Prozesse der Auslagerung militärischer Fähigkeiten von Staaten bzw. deren Verteidigungsministerien an private Sicherheitsfirmen, die als Auftragnehmerinnen von militärischen Interventionen »westlicher « Demokratien (vor allem der USA) an diversen militärisch relevanten Tätigkeiten beteiligt sind. Als kommerzielle Unternehmen handeln diese allerdings zunächst einmal ungeachtet diverser und teils dramatischer Verstöße gegen das humanitäre Völkerrecht - im Auftrag Dritter und folgen damit einer anderen Handlungs- und Funktionslogik. Insofern stellt dann die Auslagerung militärischer Fähigkeiten an den privaten Sicherheitssektor - aus Münklers Sicht die »feine und saubere Form « der Kommerzialisierung (2013: 256) - auch weniger die theoretischen Überlegungen zum Demokratischen Frieden infrage, wie Münkler behauptet (2013: 260). Im Gegenteil: Es bestätigt als Antinomie des Demokratischen Friedens eher die Notwendigkeit, die relative Friedfertigkeit von Demokratien untereinander (im Sinne der Abwesenheit zwischenstaatlicher Kriege) in einen theoretischen Bezug zu ihrem (variierenden) militärischen Außenhandeln gegenüber Nicht-Demokratien zu stellen.

Um nicht missverstanden zu werden: Typologien können und sollen nicht nur unser Verständnis über Strukturen und Wandel bestimmter politischer Phänomenbereiche (u. a. Gewalt, Frieden oder Herrschaft) erweitern, sondern unter Umständen auch die Theoriebildung voranbringen. Nur reicht es dazu nicht aus, einzelne 
Merkmalsbereiche additiv zu benennen (Privatisierung, Ökonomisierung, Brutalisierung/Asymmetrisierung). Vielmehr müssten diese wissenschaftslogisch in einen systematischen und überprüfbaren Zusammenhang über verallgemeinernde Aussagen von Wirkungszusammenhängen bzw. politisch relevanten Regelmäßigkeiten überführt werden. Wir wollen damit keinesfalls einem rigiden positivistischen Wissenschaftsverständnis huldigen. Uns ist aber eben auch bewusst, dass es gewisse Minimalstandards zur Theoriebildung geben sollte, die politikwissenschaftlich zu beachten sind - nicht zuletzt dann, wenn es sich um die Entwicklung einer empirischen Theorie handelt, die beansprucht, Aussagen über aktuelle und zukünftige politische Gewaltentwicklungen zu treffen (siehe dazu Münkler 2013: 255-257).

\section{Postkoloniale Perspektiven auf die »neuen Kriege»}

Während also die »neuen Kriege« weder in ihrer methodisch-konzeptionellen Ausrichtung noch in ihrer theoretischen Fundierung überzeugen und hinter ihrem eigenen Anspruch zurückbleiben, der Friedens- und Konfliktforschung ein neues Forschungsparadigma zu liefern, so sehr fällt auch auf, dass die Friedens- und Konfliktforschung trotz wertvoller Beiträge (Daase 2011; Geis 2006 b; Schlichte 2006) durchaus Probleme hat, der Debatte sowohl wissenschaftsintern als auch bezüglich ihrer politischen Konsequenzen adäquate Kritikperspektiven gegenüberzustellen. Postkoloniale Ansätze liefern hier eine vielversprechende Ergänzung und Erweiterung, da sie nicht nur die empirisch-methodologischen Fallstricke der »neuen Kriege« aufdecken, sondern auch zeigen, dass die »neuen Kriege« ein konflikttheoretisches Interpretationsschema liefern, das die Durchsetzung globaler Gewalt- und Herrschaftsverhältnisse begünstigt, mithin gar forciert. Anstatt sich bei der konflikttheoretischen Analyse auf eine lokale bis regionale Fokussierung einzuschränken, erweitern postkoloniale Betrachtungsweisen das analytische Spektrum der Friedens- und Konfliktforschung, indem sie Konfliktursachen nicht nur in vermeintlichen »Krisengebieten« verorten, sondern deren Querverbindungen mit und Verwurzelung in der »ersten Welt « sichtbar und benennbar machen. Postkoloniale Perspektiven müssen somit noch nicht einmal zur neuen »Lieblingstheorie« der Friedens- und Konfliktforschung erhoben werden, um zu erkennen, dass konflikttheoretische Arbeiten derzeit weit hinter den Stand vorhandener kritisch-theoretischer Überlegungen aus anderen wissenschaftlichen Disziplinen und Denkrichtungen zurückfallen. Umso auffälliger ist es, dass eine systematische Auseinandersetzung mit postkolonialen Studien in der Friedens- und Konfliktforschung kaum stattgefunden hat. Wie Bettina Engels zeigt, beschränken sich bisherige Anwendungsfelder entweder auf die Verwendung des Labels »postkolonial« als räumlich- 
historische Kategorie - des eigentlichen theoretischen und herrschaftskritischen Anspruchs postkolonialer Ansätze gänzlich entledigt -, oder aber postkoloniale Denkfiguren werden vereinzelt herangezogen, um Fragen aus den Bereichen Konflikt, Sicherheit, Krieg und Frieden zu klären (Engels 2014: 132-135). Insgesamt habe sich jedoch »das wachsende Interesse an der postkolonialen Theorie kaum in der empirischen Wissenschaft niedergeschlagen« (Engels 2014: 133; ähnlich: Ziai 2012a). ${ }^{19}$

Postkoloniale Theorien repräsentieren dabei weder ein in sich geschlossenes Theoriegebäude noch orientieren sich ihre vielfältigen analytischen und methodischen Ansätze an den gängigen Einteilungen »westlicher « Wissenschaftsdisziplinen. Einendes Merkmal ist vielmehr der Anspruch, die wechselseitigen Prozesse der Konstitution von europäischem »Selbst « und außereuropäischem »Anderen « in Bezug auf die Vergangenheit und Kontinuitäten (neo-)kolonialer Herrschaftsverhältnisse zu entschlüsseln. Das trügerische Präfix »post« darf in diesem Zusammenhang nicht dazu verleiten, das formale Ende der Kolonialzeit mit dem Verschwinden kolonialer Ungleichverhältnisse gleichzusetzen, besteht doch das Anliegen postkolonialer Ansätze gerade »in der Thematisierung des Fortbestehens und Nachwirkens einer Vielzahl von Beziehungsmustern und Effekten kolonialer Herrschaft « (Conrad/Randeria 2002: 24). Im Zusammenhang mit den Nachwirkungen kolonialer Herrschaftsverhältnisse geht es postkolonialen Ansätzen daher insbesondere darum, die diskursiven Kontinuitäten europäischer und »westlicher" Dominanz aufzuzeigen und mit dem Projekt einer umfassenden Herrschaftskritik zu verbinden. Im Mittelpunkt steht so die Kritik an einer »westlich « geprägten Wissenschaftswelt, welche im Namen eines neutralen Universalismus koloniale Wissensformen fortschreibt und vielschichtige globale Herrschaftsverhältnisse in binär kodierte Gegensatzraster (rational/irrational, entwickelt/unterentwickelt, männlich/ weiblich, modern/traditionell etc.) fasst. Der Begriff der Repräsentation, dem eine machtpolitische Dimension in Form des Schreibens über »Andere« zugesprochen wird, erlangt somit eine zentrale Stellung (Conrad/Randeria 2002; Spivak 2008; Ziai 2010). María Do Mar Castro Varela und Nikita Dhawan sprechen in Bezug auf postkoloniale Theorie dementsprechend von einem Set diskursiver Praxen, »die

19 Umso wichtiger ist daher eine fundierte institutionelle Verankerung postkolonialer Forschung und Lehre an Universitäten sowie eine verstärkte Verschränkung konflikttheoretischer und postkolonialer Forschungsarbeit (Engels 2014; Ziai 2012 a). Beides sollte dazu beitragen, einer von akademischen Modeerscheinungen abhängigen Einbeziehung postkolonialer Perspektiven entgegenzuwirken, die der Gefahr einer Kommodifizierung und Vereinnahmung postkolonialer Theorie durch »Erste-Welt-Intellektuelle« letztlich nicht entgehen kann (Castro Varela/Dhawan 2009: 10). 
Widerstand leisten gegen Kolonialismus, kolonialistische Ideologien und ihre Hinterlassenschaften« (2005: 25).

\subsection{Privatisierung und failed states}

Als eine solche Hinterlassenschaft muss aus postkolonialer Sicht das Konzept sogenannter failed states ${ }^{20}$ betrachtet werden. Der Forschungsstrang der failed states, dem im Zuge des war on terror eine zunehmend wichtige Rolle eingeräumt wird - etwa bei der Beschreibung und Konstruktion terroristischer »Rückzugs- und Gefahrenräume« -, zielt in erster Linie darauf, Prozesse des Staatszerfalls zu erkennen bzw. vorherzusagen, indem Funktionsfähigkeit, Ordnungs- und Wohlfahrtsleistungen von Staaten vergleichend erfasst und bewertet werden. ${ }^{21}$ Als failed states gelten demnach jene Staaten, welche weder ein territoriales Gewaltmonopol noch effektives wie legitimes Herrschaftshandeln aufrecht erhalten können (Goldstone 2008: 286) bzw. darüber hinaus nicht in der Lage sind, grundlegende Sicherheitsleistungen und Wohlfahrtsgüter zur Verfügung zu stellen (Rotberg 2007: 83; 2004: 1-10; Schneckener 2004: 14-17). Von Seiten der Friedens- und Konfliktforschung wurde eine solche Ausrichtung dahingehend kritisiert, eine Idealisierung europäischer Staatlichkeit zu betreiben (Daase 2011: 23-24), die sich zu einem handfesten Wahrnehmungsproblem »westlicher« Wissenschaft verdichtet, wenn Ordnungsstrukturen abseits europäischer Staatsvorstellungen aus Analyserastern ausgeschlossen werden (Geis 2006 b: 14-17). Eine solche Kritikperspektive ist durchaus anschlussfähig an postkoloniale Beiträge, die der These der failed states in nahezu all ihren Varianten ein eurozentristisches Staatsideal - angelehnt zumeist an Max Webers Idealtyp-Formel aus Gewaltmonopol, Staatsvolk und Staatsterritorium (Weber 1988; kritisch: Wai 2012)22 - nachweisen bzw. das Konzept der failed states als einen selbstversichernden Herrschaftsdiskurs »des Westens« erkennen (Hill 2005). Eine tiefer gehende Auseinandersetzung mit den eurozentristischen Schieflagen der Debatten um failed states ist die Friedens- und Konfliktforschung dennoch schuldig geblieben. Postkoloniale Ansätze können diese

20 Diverse Abweichungen in den verwendeten Begrifflichkeiten - etwa die Bezeichnung collapsed state anstelle von failed state - kommen zwar vor, spielen für unsere Argumentation jedoch eine untergeordnete Rolle. Aus diesem Grund greifen wir einheitlich auf den Begriff der failed states zurück.

$21 \mathrm{Zu}$ den gesellschaftstheoretischen und politischen Problematiken raumbezogener Vergleiche vgl. Belina/Miggelbrink (2010).

22 Auch wenn in der Literatur zumeist auf Webers Staatsdefinition aus »Politik als Beruf« aus dem Jahr 1919 verwiesen wird, ist anzumerken, dass schon Georg Jellinek (1900) auf eine Staatsdefinition entlang der Trinität Staatsgebiet, Staatsvolk sowie Staatsgewalt zurückgreift. 
Leerstelle füllen, indem sie erstens auf die parallele Historizität (post-)kolonialer Gewaltverhältnisse und »westlicher « Wissensproduktion aufmerksam machen und zweitens, ausgehend von diesem erweiterten Verständnis, alternative Perspektiven auf Prozesse vermeintlichen »Staatszerfalls« entwickeln, die den Problemhorizont auf die machtvollen Verstrickungen und politischen Einflussnahmen ehemaliger Kolonial- oder heutiger Großmächte erweitern.

Im Fokus einer postkolonialen Kritik am Konzept der failed states steht in allererster Linie der Nexus zwischen europäischem Kolonialismus und »westlicher" Wissensproduktion. Die Etablierung von Fächern wie Anthropologie, Sinologie oder Indologie im Europa des 19. Jahrhunderts und die damit verbundene Grenzziehung zwischen dem »Wissenssubjekt « Europa und den »Wissensobjekten« der "Anderen« stand demnach keineswegs allein im Dienste der Wissenschaft, sondern in ebenso engem Zusammenhang mit dem europäischen Imperialismus, der durch die orientalistische Wissensproduktion »westlicher « Intellektueller wissenschaftliche Legitimation erfuhr(Bilgin/Morton 2002: 57-62; Conrad/Randeria 2002: 20-22; grundlegend: Said 2009). ${ }^{23}$ Entsprechend spricht Zubairu Wai im Zusammenhang mit der Genese postkolonialer Staatlichkeit von einer

"co-constitutive, almost parasitic, relationship between states in the West and the so-called Third World, which were constituted under concrete conditions of Western colonial domination and which have remained immersed in the politics of global economic and sociopolitical structures of unequal power relations that produce wealth and affluence on the one hand and conditions of dependence and insecurities on the other « (2012: 39).

Da der Begriff der failed states jedoch fernab der Historizität (post-)kolonialer Gewalt und Wissensmacht operiert, kann er die Kontinuitäten, Brüche und Widersprüche im Übergang von je verschiedenen kolonialen Herrschaftsformen ${ }^{24} \mathrm{zu}$ den wiederum vielschichtigen Realitäten heutiger postkolonialer Staaten nicht einmal ansatzweise in den Blick bekommen. Er bleibt damit sowohl gegenüber der Vergangenheit und Gegenwart globalpolitischer Macht- und Beziehungsgeflechte als auch den damit verbundenen Transformationsprozessen erschreckend unterkomplex. In Abgrenzung zu solchen Verkürzungen bemühen sich postkoloniale Kritik-

23 In spiegelbildlicher Analogie wurde damit auch Europa zum Objekt der Wissenschaften - allerdings im Namen »universaler« Werte sowie »universalen« Wissens, dessen Abgrenzung vom »Partikularwissen « außereuropäischer Wissensgebiete sich bis heute in den disziplinären Einteilungen der »westlichen« Wissenschaftswelt widerspiegelt (Conrad/Randeria 2002: 20-22).

24 Castro Varela und Dhawan (2005: 12-13) unterscheiden in Anlehnung an Osterhammel (2003) zumindest grob zwischen den drei Formen der Beherrschungs-, Stützpunkt- und Siedlungskolonie. 
perspektiven darum, jene historischen Kontinuitäten offenzulegen, die den politischen und sozio-ökonomischen Rahmen für Ereignisse wie die gewaltsame Transformation von Staatlichkeit erst eröffnen.

Eine dezidiert postkoloniale Alternative zum Begriff der failed states liefert Shalini Randeria mit ihrem Konzept des »listigen Staates « (Randeria 2009; Dhawan 2009: 59). Aus diesem Blickwinkel erscheinen außereuropäische Staaten nicht »als >schwach schichte " (Randeria 2009: 214). Nicht nur den Thesen der »neuen Kriege«, auch einer weltgesellschaftlichen Orientierung ist Randeria damit in zweifacher Hinsicht voraus: Zum einen verweist sie explizit auf die historische Spezifik postkolonialer Staatlichkeit. Entsprechend begreift sie den »listigen Staat« als gewieften Akteur, der zwar den neoliberalen Reformprogrammen internationaler »Geberinstitutionen « (Welthandelsorganisation, Internationaler Währungsfonds und Weltbank) unterworfen ist, gleichzeitig jedoch diese Beschränkungen sowohl gegenüber der internationalen Gemeinschaft als auch gegenüber der eigenen Bevölkerung auszuspielen weiß - und sich so Handlungsspielräume offen hält. Die Strukturanpassungsprogramme der Weltbank werden so als nahtlose Fortsetzung kolonialer Beherrschungsstrategien begreifbar (Randeria 2009: 215), deren Rückwirkungen auf akteurs- und strukturgebundene Dynamiken im Krieg eine analytische Herausforderung für die Friedens- und Konfliktforschung darstellen. Zum anderen betont Randeria (2009: 226) die nach wie vor nicht zu unterschätzende Bedeutung staatlicher Handlungsmacht bei der Durchsetzung und Regulierung von Globalisierungsprozessen. Sie widerspricht somit dem weltgesellschaftlichen Paradigma einer Entgrenzung der Welt, das die postkolonialen Beziehungsgeflechte zwischen Europa und außereuropäischen Gebieten und ihre konstituierende Rolle für die Moderne hinter einer abstrakten Totalität der Welt verschwinden zu lassen droht (Con$\mathrm{rad} /$ Randeria 2002: 18).

Im Umkehrschluss gilt dann wiederum nicht, dass postkoloniale Perspektiven Akteur_innen unterhalb der staatlichen Makroebene aus ihrem Blickfeld verlieren. So bieten sie gerade den Vorteil, sich nicht starr an klassisch soziologischen Akteursebenen zu orientieren (staatliche, gesellschaftliche sowie individuelle Ebene), sondern von diskursiven und materiellen Macht- und Wissensstrukturen auszugehen, die je verschiedene Akteursebenen miteinander verbinden und in Beziehung setzen - womit ebenso die Realität nichtstaatlicher Ordnungen wie die des »listigen Staates « in den Analysefokus rückt. Insofern darf die postkoloniale Betonung der Regulierungsmacht von Staaten nicht als Affirmation staatlicher Herrschafts- und Unterdrückungstendenzen missinterpretiert werden, die sich gar mit den Überlegungen der failed states in Übereinstimmung bringen ließe: Es sind ganz offen- 
sichtlich zwei verschiedene Dinge, eine postkoloniale Kritik an der Unterschätzung staatlicher Handlungsoptionen zu formulieren und postkoloniale Staaten vor dem Hintergrund eines eurozentristischen Idealbildes politisch und militärisch angreifbar zu machen.

Vielmehr erschließen postkoloniale Ansätze eine wertvolle konflikttheoretische Alternative zu den verkürzenden territorialstaatlich orientierten Deutungsmustern der failed-state-These. Denn so wie diese das Phänomen auf einer nationalen Raumebene (scale) fixiert und damit Erklärungszusammenhänge, die erst auf globaler Ebene sichtbar werden, strukturell ausschließt, verdeckt eine Ausklammerung globaler postkolonialer Machtverhältnisse zugleich die historisch-spezifischen Entstehungsbedingungen postkolonialer Staatlichkeit. Das Konzept der failed states unterliegt damit einem Phänomen, das der Humangeographie längst als politics of scale bzw. scale jumping (Smith 1987; 1990; Wissen 2008) bekannt ist und mittels der gezielten Ausblendung alternativer skalarer Ebenen, je nach Erfordernis entweder »das Lokale« oder »das Globale« zur alleinigen Ursache sozialen (Konflikt-)Verhaltens stilisiert (Belina 2013: 104). Eine raum- und konflikttheoretisch informierte postkoloniale Perspektive hingegen öffnet neue Analysewege: Anstatt das Phänomen der failed states auf eine nationale oder eine globale scale einzuengen, lässt es sich in Bezug auf und zwischen verschiedenen Raumformen (territory, place, scale, networks) und deren materiellen sowie symbolischen Konstituierungsmechanismen untersuchen (Belina 2013: 86-133; Jessop et al. 2008). Schließlich lassen sich erst mit raumtheoretischen Konzepten wie place-making und territoriality die sich wechselseitig verstärkenden konstitutiven Dimensionen materieller Raumproduktion entlang politischer Herrschaft, Gewalt und Territorium (Grenzziehung) aufzeigen und in Verbindung zu diskursiven und symbolischen Wert- und Bedeutungszuschreibungen räumlicher Bezüge untersuchen. Gerade weil diese für kollektive Akteur_innen identitätsstiftend sind und raumbezogenem Konflikthandeln Sinn geben, bieten sie auch einen Ansatzpunkt, die variierenden Gewaltdynamiken innerhalb von Kriegen oder zwischen verschiedenen Kriegen (und Kriegstypen) zu erklären (u. a. Kalyvas 2006; Tilly 2003; Weinstein 2007).

\subsection{Postkoloniale Perspektiven auf Ökonomisierung und Entpolitisierung}

Ein eurozentristisches Staats- bzw. Politikideal macht sich nicht allein in Bezug auf das problematische Konzept der failed states bemerkbar. Auch in Zusammenhang mit dem Argument einer vermeintlichen Ökonomisierung der »neuen Kriege« verhindert die Vorrangstellung des »westlich«-idealisierten Staates einen ausgewogenen Blick auf Prozesse der (Ent-)Politisierung von Gewalt im Allgemeinen 
und Krieg im Speziellen. Konflikttheoretische Beiträge haben dieses Vorgehen in zweifacher Hinsicht kritisiert: Die »neuen Kriege«, so der Vorwurf, bleiben erstens blind für politische Konstellationen außerhalb der »westlichen Welt« (Schlichte 2006: 117-119) und vernachlässigen zweitens die politischen Motivlagen nichtstaatlicher Gewaltakteur_innen (Daase 2011: 24-25). Damit ist das Problem zwar tatsächlich richtig benannt und ebenso scharf umrissen. Bislang sind jedoch, so unsere These, die postkolonialen Erklärungsangebote zur kritisch-konstruktiven Bearbeitung dieser - durchaus weitreichenden - wissenschaftlichen Schieflage bei Weitem noch nicht ausgereizt worden.

Postkoloniale Theorien nehmen doch gerade diejenigen Wissensbestände europäischer politischer Theorie kritisch in den Fokus, die den Begriff des Politischen spätestens seit Thomas Hobbes unweigerlich an den modernen europäischen Nationalstaat knüpfen (Ziai 2012 a: 285). Die Setzung des Staates als Dreh- und Angelpunkt europäischer Politikvorstellungen und die damit einhergehende Gleichsetzung von »Politik« mit staatlichen Ordnungsvorstellungen führte im Kontext europäischer Kolonialeroberungen nicht nur dazu, dass vorgefundene politische Organisationsformen als »vorzivilisatorisch« verworfen wurden. Politische Systeme afrikanischer Gesellschaften wurden darüber hinaus ebenso aus den Wissensarchiven europäischer Geschichtsschreibung getilgt (Ayers 2006; Feierman 2002) wie auch außereuropäische politische Ereignisse globaler Reichweite, etwa die Haitianische Revolution von 1791, kaum Beachtung fanden und bis heute marginalisiert bleiben (Grovogui 2006; Trouillot 2002). Aram Ziai spricht dementsprechend von einer europäischen Theorietradition, die die Vorrangstellung des Staates als "grundlegende politische Gemeinschaft und Einheit des Politischen« (2012a: 285) bis heute fortschreibt.

Eines der zentralen Anliegen postkolonialer Theorie besteht folglich darin, die machtverwobene Historizität und Selektivität »westlicher « Wissensbestände sichtbar zu machen, welche sich von »westlichen« Akteur_innen in normativ aufgeladene Dichotomien (hier: »politisch vs. unpolitisch«) übersetzen lassen. Dipesh Chakrabarty beschreibt eine solche »Provinzialisierung « Europas als »Geste der Umkehrung « (2002: 289), der es darum geht, die verfestigten Metaerzählungen universeller Geschichtsschreibung zu durchbrechen. Das Ziel, Europa als Referenzund Endpunkt einer universalen Entwicklungsgeschichte zu entmythisieren und Ordnungsstrukturen jenseits europäischer Staatsmodelle in den Bereich des Politischen zu re-integrieren, setzt freilich zunächst die Erkenntnis voraus, dass modernes Denken, der Anspruch auf Wissenschaft und Rationalität und somit auch moderne Vorstellungen des Politischen nicht auf europäischem Boden allein gewachsen sind. Vielmehr können diese Entwicklungen nur aus dem Spannungsverhältnis zwischen 
Europa und »seinen« ehemaligen Kolonien heraus erklärt werden (Chakrabarty 2002: 303-304). Insofern geht es postkolonialer Theorie um die Etablierung einer "geteilten Geschichte «(Conrad/Randeria 2002: 17-22), die im Sinne der englischen Doppelbedeutung des Wörtchens "geteilt« (shared/divided) verstanden werden muss: Einerseits also im Sinne einer shared history, die den Austausch zwischen europäischen und außereuropäischen Erfahrungen in den Vordergrund rückt und Europas Alleinanspruch auf die tiefgreifenden Umwälzungen der Moderne zugunsten eines relationalen Geschichtsbegriffes verwirft; andererseits jedoch auch im Sinne einer divided history, welche die machtpolitischen Ungleichheiten und gewaltvollen Repressionen, die mit der wechselseitigen Beziehung zwischen »westlicher « und »nicht-westlicher« Welt einhergingen und -gehen, betont (etwa Fanon 1981). ${ }^{25}$ In den Worten Chakrabartys: »Es geht darum, in die Geschichte der Moderne die Ambivalenzen, die Widersprüche, die Gewaltanwendung und die Tragödien und Ironien einzuschreiben, die sie begleiten « (2002: 306).

Postkoloniale Theorien eröffnen somit mindestens zwei erweiternde Sichtweisen auf die Streitfrage einer angeblichen Depolitisierung der »neuen Kriege«. So ist erstens das, was von »westlichen« Wissenschaftler_innen heute als politisches Konflikthandeln interpretiert wird, aus postkolonialer Sicht nicht nur ein Ergebnis individueller Entscheidungen - »welche Theoriebrille wollen wir uns aufsetzen?« -, sondern vielmehr auch ein Aspekt, der auf wissensstruktureller Ebene verhandelt wird. Nehmen wir den postkolonialen Einwand ernst, dass Wissens- und Machtordnungen schwerlich voneinander zu trennen sind und Perspektiven außerhalb normierter Vorstellungen von $»$ Rasse $\aleph^{26}$ und Geschlecht auch im heutigen akademischen Betrieb vor erheblichen Hürden stehen (Kilomba 2008: 25-38), dann bedarf es zweitens einer tief gehenden Auseinandersetzung mit den wissensstrukturellen

25 Aus Sicht einer geteilten Geschichte kann folglich einerseits Münklers Annahme, der Unterschied zwischen den Staatsbildungskriegen Europas bzw. Nordamerikas und den Staatszerfallskriegen in der »dritten Welt« bestünde hauptsächlich darin, dass »Erstere unter quasi-klinischen Bedingungen, also ohne größere Einflüsse `von außen`, verlaufen sind« (2002: 18) als Idealisierung der Genese europäischer Staatlichkeit enttarnt werden, deren Verstrickungen in die Gewalttaten und ökonomischen Bereicherungen des Kolonialismus ausgeklammert werden. Andererseits wird deutlich, dass Münklers Konzept heroischer bzw. postheroischer Gesellschaften die Tradition kolonialistisch-dichotomer Welteinteilungen (»Erste Welt« vs. »Dritte Welt«, »Moderne« vs. »Tradition«) fortsetzt, indem es eine natürlich gegebene und trennscharfe Grenzziehung zwischen »westlichen« und »nicht-westlichen« Gesellschaften suggeriert (2010: Kap. 16).

26 Wir verwenden an dieser Stelle bewusst den Begriff »Rasse«, um die Gewalttätigkeit (konstruierter und erfundener) $»$ Rassevorstellungen $«$ auch sprachlich abzubilden. Die Verwendung des englischen Terminus race ist in den deutschen Critical Whiteness Studies ebenfalls gebräuchlich. Für einen Überblick und eine kritische Diskussion beider Begriffe vgl. Wollrad (2005) sowie für eine Einordnung des Konzepts »Rasse« in kolonialistische Herrschaftsdiskurse Arndt (2011). 
und machtverwobenen Hinterlassenschaften mehrerer Jahrhunderte kolonialer Herrschaft - in der Friedens- und Konfliktforschung sowie darüber hinaus. Obwohl kritische Veröffentlichungen innerhalb der Friedens- und Konfliktforschung (siehe v. a. Daase 2011; Schlichte 2006) durchaus in die richtige Richtung weisen, sind wir überzeugt, dass selbst diese Ansätze von einer expliziteren Auseinandersetzung mit den Wissensbeständen postkolonialer Theorie profitieren würden und damit der konflikttheoretischen Herausforderung, die verbindenden wie auch widersprüchlichen Tendenzen einer "geteilten Geschichte« auf ihre konflikttheoretischen Implikationen hin zu untersuchen, weitaus besser gewachsen wären. Eine postkoloniale Perspektive kann nicht zuletzt zeigen, dass erst in Auseinandersetzung mit einer »westlichen« Theorietradition jene historischen und wissenstheoretischen Bedingungen sichtbar werden, unter denen Staaten den Status als politisch anerkannte Akteure gewinnen oder verlieren - und gleichzeitig nichtstaatlichen Gewaltakteur_innen Legitimität zu- oder abgesprochen wird. Hier wird noch einmal deutlich, dass die Friedens- und Konfliktforschung nicht nur mit einem Wahrnehmungs- und Kompetenzproblem konfrontiert ist, sondern auch einem Deutungsproblem gegenübersteht. Letzteres offenbart sich vor allem dann, wenn kolonialistische Wissensbestände die sprachliche Einrahmung empirischer Konfliktanalysen prägen. ${ }^{27}$

\subsection{Postkoloniale Perspektiven auf Brutalisierung}

So verzerrt die Wahrnehmungs- und Deutungsbilder der »neuen Kriege« hinsichtlich von failed states und von Prozessen der Entpolitisierung (Ökonomisierung) sind, so einseitig wird auch das Diskussionsfeld der Brutalisierung beleuchtet. Wir erinnern uns: Im Mittelpunkt steht hier die These einer Ausweitung und Intensivierung (sexualisierter) Gewalt in den »Massakern« »neuer Kriege«, die mit der vermeintlichen Einhegung von Gewalt in den europäischen »Staatsschlachten « kontrastiert wird (Münkler 2002: 144). Wenn die Frage von Gewaltanwendung im All-

27 Eine postkolonial informierte Friedens- und Konfliktforschung könnte hier dazu beitragen, kolonialistische Stereotype zu vermeiden, wie sie beispielsweise im Zuge der anhaltenden Kämpfe um territoriale Kontrolle in Mali zu beobachten waren. Wenn beispielsweise Lecocq et al. (2013 a: 16, 22) in ihrer Analyse Mali wiederholt als verwundetes Nilpferd bezeichnen, das hilflos durch trübe Wasser treibe, ist der Schritt zu einer Krankheits- und Tiersprache, wie sie schon Frantz Fanon (1981:35-36) als Teil kolonialistischer Herrschaftsstrategien benannte, nicht weit. Ähnlich sensibel sollte dann auch mit diskursiven Setzungen wie »Mali: auf dem Weg zu einem `Sahelistan «? « (Ehrhart 2013: 251) umgegangen werden, bei der zwei räumlich und zeitlich disparate Gewaltereignisse - die Kriege in Afghanistan bzw. Mali - ihren spezifischen Kontexten entrissen und einem europäischen »Selbst« gegenübergestellt werden, das Kriege außerhalb des »globalen Nordens« simplifizierend unter die Kategorie »nicht-westlich« subsumiert. 
gemeinen und sexualisierter Gewalt ${ }^{28}$ im Besonderen sowohl von Münkler (2013; 2002: 142-153) als auch von anderweitig orientierten Vertreter_innen der »neuen Kriege « wiederholt betont wird (Heupel/Zangl 2004), dann muss aus postkolonialer Sicht zunächst noch einmal herausgestellt werden, dass Gewalt nicht nur physisch in Erscheinung tritt, sondern immer auch auf diskursive Gewaltverhältnisse Bezug nimmt und an diese anknüpft (Brunner/Hrzán 2009: 101-102). Postkoloniale Ansätze zielen hierbei darauf ab, jene Formen epistemischer Gewalt (Spivak 2008) sichtbar zu machen, die »westlichen « Akteur_innen vor dem Hintergrund rassifizierter, vergeschlechtlichter, patriarchaler und heterosexualisierter Binarisierungen Handlungsspielräume eröffnen und ihnen zugleich als Legitimation eines militärischen Eingreifens dienen.

Vor dem Hintergrund dieser postkolonialen Perspektiverweiterung lassen sich dann durchaus parallele Argumentationslinien in historisch wirkungsmächtigen Kolonialdiskursen und den »neuen Kriegen« nachverfolgen. In beiden Fällen nämlich wird auf die Figur der »unterdrückten anderen Frau « (Castro Varela/Dhawan 2009: 11) rekurriert, deren Rettung als Bürde des weißen Mannes bzw. der »westlichen « Staatenwelt ausgegeben wird. ${ }^{29}$ Eine zentrale These der »neuen Kriege« besteht folgerichtig darin, dass sexualisierte Gewalt gegen Frauen vor allem mit Kriegen jenseits des Staates in Verbindung zu bringen sei (Münkler 2002: 142-153; 2010: 300). Diese Annahme, die durch die Kontrastierung der »Organisationsrationalität« staatlicher Streitkräfte (Münkler 2002: 148) mit der kulturalistischen und teils biologistischen Beschreibung exzessiver Gewaltanwendung bei paramilitärischen Akteur_innen zusätzlich hervorgehoben wird (Münkler 2002: 151-152), rückt die »neuen Kriege« in gefährliche Nähe zu Kolonialnarrativen. Diese nutz(t)en ein entlang rassifizierter und heterosexualisierter Pauschalisierungen konstruiertes orientalisches Patriarchat als Legitimationsfolie politischen und militärischen Handelns. So diente aus historischer Sicht schon die Praxis des sati, der erzwungenen Witwenverbrennung in Indien, britischen Kolonialherren (sic!) dazu, kolonialistische Machtbestrebungen mit dem Scheinargument der Errettung indischer Frauen vor ihren gewalttätigen Männern zu rechtfertigen (Castro Varela/Dhawan 2009: 11-13; Spivak 2008). Ebenso verdeutlichen Arbeiten, die sich aus feministischpostkolonialer Sicht mit Formen terroristischer Gewalt beschäftigen (etwa Brunner 2011; Thomas/Virchow 2010), dass heterosexualisierte und rassifizierte Legitima-

28 Grundlegend für die Thematisierung von Gewalt gegen Frauen und insbesondere die Beschreibung von Vergewaltigungen als (männliche) Waffe im Krieg: Brownmiller (1975).

29 Vgl. in diesem Zusammenhang etwa die Stellungnahme von Aminata D. Traoré (2013) zur Intervention Frankreichs in Mali, welche Staatspräsident Hollande unter anderem mit Verweis auf die Rechte und die Unterdrückung malischer Frauen legitimierte (L'Express 2013). 
tionslogiken nicht von globalen Machtverhältnissen zu trennen sind. Claudia Brunner und Daniela Hrzán (2009) zeigen hierbei, wie die »westlichen« Diskurse um Female Suicide Bombing und Female Genital Cutting, so widersprüchlich die den »nicht-westlichen« Frauen hier zugewiesenen Subjektpositionen auch sein mögen (Täterinnen vs. Opfer), von der kulturalisierenden ${ }^{30}$ Vorstellung eines orientalistischen Patriarchats zusammengehalten werden. Auch wenn die historischen Ausgestaltungen »westlicher « Legitimationsfiguren somit durchaus variieren, tragen postkolonial-feministische Arbeiten explizit dazu bei, jene Kontinuitäten aufzudecken, die viktimisierte orientalisch-weibliche Subjekte als Legitimationsfolie eines »westlichen« Interventionsdiskurses (re-)konstruieren. Vor diesem Hintergrund sind die Thesen der »neuen Kriege« dann nicht nur in Hinblick auf ihre analytischen Verkürzungen und kulturalisierenden Tendenzen konflikttheoretisch problematisch, sondern auch friedenspolitisch in dem Maße gefährlich, in dem sie militärische Intervention als notwendige Strategie der Konfliktbearbeitung nahelegen: »Unter solchen Umständen kann dem Kriegsvölkerrecht allenfalls von außen durch die bewaffnete Intervention eines Dritten Geltung verschafft werden« (Münkler 2002: 148).

Dass die vielfältigen Beziehungen zwischen Krieg, Geschlecht und Medien sich dabei selbst wechselseitig verstärken und politisch-militärischen Kraftfeldern ausgesetzt sind, legen, wie in Abschnitt 2.3 bereits angedeutet, Beiträge aus dem Bereich der kritischen Medienforschung nahe. Diese analysieren, wie die mediale und insbesondere bildliche Repräsentation der zu befreienden/bekämpfenden »Anderen « stereotype Wahrnehmungsmuster verstärken, wenn diese - je nach Erfordernis - als weiblich-viktimisierte Opfer oder auch als barbarisch-männliche Kämpfergestalten gezeichnet werden (Maier/Balz 2010). ${ }^{31}$ Elisabeth Klaus und Susanne Kassel (2008) zeigen zudem, dass nicht nur die bipolare Darstellung weiblicher und männ-

30 Unter »Kulturalisierung « verstehen Brunner und Hrzán Prozesse, »in deren Rahmen unterschiedliche soziale Phänomene auf wenige prägnante Merkmale reduziert und somit miteinander unter diesem gemeinsamen Nenner kompatibel gemacht werden können« (2009: 96). Einem solchen Vorgehen entspricht ein statisch gefasster Kulturbegriff, der in komplexitätsreduzierender und essenzialisierender Weise nicht-europäische Subjekte auf ihre Rückständigkeit und Unveränderbarkeit hin fixiert.

31 In ähnlicher Weise macht Krista Hunt (2006) mit ihrem Konzept des embedded feminism aufmerksam auf die Instrumentalisierung von Frauenrechten zur Kriegslegitimation im Allgemeinen und die problematische Rolle »westlich«-liberaler Feminist_innen bei der Legitimierung des USamerikanischen war on terror im Besonderen. Iris M. Young (2003) verweist darüber hinaus auf die vergeschlechtlichte Dimension »westlichen« Staatshandelns, das sich der Rolle ritterlich-beschützender Männlichkeit bedient, um sowohl Sicherheitsverschärfungen nach innen (weiblich konnotierte, zu beschützende Bürger_innen) als auch eine militärische Politik nach außen (aggressive Maskulinität der »Anderen«) zu legitimieren. 
licher Stereotype im Krieg auf eine lange Geschichte zurückblicken kann (Geschlechterlogik) und die Konstruktion aufwertender Selbst- gegenüber abwertender Fremdbilder seit jeher Bestandteil kriegerischer Auseinandersetzungen sind (Kriegslogik). Darüber hinaus verweisen sie auf eine »strukturelle Militarisierbarkeit« (Klaus/Kassel 2008: 269) der Medien, die bewusst mit dichotomen Zuspitzungen arbeiten, um zugleich Aufmerksamkeit und Auflage zu vergrößern. Ihre Feststellung, dass die in Medien zur Instrumentalisierung von Frauenrechten verwendeten Bilder durchaus variieren, Darstellungen der »anderen « Frau jedoch immer wieder das Klischee der Opferrolle bedienen, ${ }^{32}$ deutet zum einen auf die Anpassung medialer und militärischer Legitimierungsstrategien an breitere diskursive Rahmen(-setzungen) hin (Butler 2009: 32). Zum anderen wird aber auch die Beständigkeit deutlich, mit der stereotype und kolonialistische Bilder der »Anderen « in mediale Diskurse und Bildrepräsentationen eingehen und über die produzierten »dichotomen und wertenden Abgrenzungszuschreibungen« sowie die »Kopplung von Kulturen bzw. Religionen an bestimmte Räume« (Strüver 2010: 91) zur Kriegslegitimation beitragen.

Um diesen Punkt noch einmal zu akzentuieren: Wenn lokale Akteur_innen sowohl in wissenschaftlichen als auch in medialen Repräsentationen weithin nur entlang der Dichotomie »viktimisiertes weibliches Subjekt« vs. »barbarisch-männliches Kämpfersubjekt « erfasst und noch dazu in einen analytischen Zusammenhang mit den Folgeerscheinungen kriegerischer Gewalt und Flucht gesetzt werden (Münkler 2002: 31), liegt die Schlussfolgerung nahe, dass allein »westliche« Kräfte für eine effektive Bearbeitung von gewaltsamen Krisen infrage kommen. Und weitergedacht: Da dann als Adressaten eines state-building in der Folge militärischer Interventionen meist nur die alten politischen Eliten als Verhandlungspartner vorstellbar erscheinen, bleiben Frauen (oder andere Geschlechteridentitäten) im Rahmen »westlicher" Viktimisierungsdiskurse als selbstbestimmte und politisch aktive Subjekte von den staatsbildenden Prozessen ausgeschlossen. Mechthild Exo $(2009 ; 2011)$ verweist in diesem Zusammenhang auf politische Basisbewegungen und die feministische Opposition in Afghanistan, ${ }^{33}$ deren politische Arbeit vom dominanten Narrativ der unterdrückten muslimischen Frau weitgehend unsichtbar

32 In den Kriegen in Bosnien und im Kosovo etwa durch das Bild flüchtender Frauen, im Afghanistankrieg hingegen in Form der »entschleierten orientalischen Frau« als Sinnbild für die Befreiung durch »westliche« Mächte (Klaus/Kassel 2008: 269-276).

33 Beispielhaft sei hier vor allem auf die Revolutionary Association of the Women of Afghanistan (RAWA) verwiesen, die selbst mehrere Stellungnahmen zur anhaltenden Konflikteskalation durch "westliche« Mächte auf der einen und fundamentalistische Terrororganisationen auf der anderen Seite verfasst hat (etwa RAWA 2008). 
gemacht wird. Deutlich werden sollte damit, dass eine postkolonial informierte Friedens- und Konfliktforschung globale wie nationale Diskurs- und Machtkonstellationen mit lokalen Akteurs- und Handlungsebenen in Verbindung setzen muss, will sie die bereits erkannten Verzerrungen im eigenen Wahrnehmungsbild vermeiden.

Ebenso verlangen die zunehmende mediale Präsenz des Krieges, seine politisch motivierte Darstellung bzw. auch seine gewollte Unsichtbarmachung konflikttheoretischen Analysen gesteigerte Aufmerksamkeit im Hinblick auf die mediale Resonanz ihrer Arbeiten ab. Wenn wir daher mit Butler (2009) davon ausgehen, dass die mediale und wissenschaftliche Rahmung des Krieges einen diskursiven Richtungssinn erzeugt, der die moralische Bewertung von und die Entscheidung zu Gewalt maßgeblich beeinflusst, dann ist von konflikttheoretischer Seite umso mehr auf einen sorgsamen Umgang mit postkolonialen Machtverhältnissen zu achten, die sich entlang der oben beschriebenen Binarisierungen manifestieren. Wer hingegen den Krieg außerhalb »westlicher « Vergesellschaftungsformen weiterhin als rein lokales Phänomen und somit nur als »Dauerzustand gesellschaftlicher Selbstzerstörung" deutet (Münkler 2013: 255), übersieht zum einen die Interessen und das Agieren externer Akteur_innen, die das Feuer vieler Kriege der Gegenwart am Lodern halten. Zum anderen liefern verengte konflikttheoretische oder populärwissenschaftliche Deutungen eben auch bedenkliche mediale Anreize, herrschaftsaffirmierende Abgrenzungsdiskurse aufzugreifen, fortzuführen oder gar zuzuspitzen.

\section{Fazit}

Die hier gewählten Perspektiven postkolonialer Kritik sollten in grundsätzlicher Absicht zunächst noch einmal deutlich machen, dass die Rede von den »neuen Kriegen « die bereits im kolonialen Diskurs und in »westlicher « Wissensproduktion angelegte binäre Kodierung des europäischen »Selbst« vs. der »hilfsbedürftigen Anderen « in ihren failed states fortschreibt und dabei an patriarchalen, heteronormativen und orientalisierten Geschlechterperspektiven und Identitätskonstruktionen des methodologischen Nationalismus festhält. Auch wird mit der Geopolitisierung und Kulturalisierung von Gewalt die von der Politikwissenschaft längst als problematisch erkannte Stereotypisierung orientalisierter und geschlechtlicher Rollen reproduziert. Konflikttheoretisch ist das zum einen deswegen relevant, weil erst die gewaltvollen Verstrickungen der Kolonialmächte im Zuge der »eigenen« europäischen Staatenbildungsprozesse Ansatzpunkte dafür liefern, innerhalb welcher materiell-politischer Ausgangsbedingungen lokale und transnationale Konflikte ausgetragen werden. Neben der Einbeziehung der Historizität kolonialer Ge- 
waltverhältnisse geht es zum anderen darum, die gegenwärtigen strukturellen und diskursiven Verbindungen zwischen der Anwendung organisierter Gewalt diverser staatlicher und nichtstaatlicher Konfliktakteur_innen im »globalen Süden« und den politischen und ökonomischen (Interventions-)Motiven machtvoller staatlicher Akteur_innen und internationaler Institutionen in der »ersten Welt « einzubeziehen und offenzulegen. Konflikttheoretisch lautet die daraus resultierende Schlussfolgerung, dass gesellschaftlich-strukturelle Gewaltursachen über raum- und identitätszuschreibende (diskursive) Praktiken vermittelt und nicht nur in hermetisch geschlossenen Räumen der »Anderen« verankert sind, die allenfalls über globalisierte Kriegswirtschaften und aus den Kriegen resultierenden Flüchtlingsbewegungen mit dem »globalen Norden « verbunden sind (siehe Kaldor 2013). Die Ursachen und Dynamiken heutiger Kriege haben eben auch vielfältige Verbindungen zur »ersten Welt « und sind in regionale wie globale Machtstrukturen eingebettet bzw. mit der »Moderne « verwoben (Randeria 1999).

Der Beitrag mahnt dann auch einen skeptischen Umgang mit jenen Analysen an, die dem Deutungsmuster der "neuen Kriege « bewusst oder unbewusst folgen und Gewaltereignisse jenseits »westlicher « Staatlichkeit lediglich als Folgen »schwacher staatlicher Autorität«, »Korruption« und der Durchsetzung mit »kriminellen Netzwerken« interpretieren oder gar »aus der Kolonialzeit stammende Ressentiments gegenüber Europäern « als bestimmende (und regional begrenzte) Konfliktursachen anführen (Pabst 2013: 1-2; ähnlich: Cline 2013; Lecocq et al. 2013 b; Lounnas 2013). ${ }^{34}$ Dagegen bieten differenziertere Ansätze der Friedens- und Konfliktforschung mit ihrer kritischen Einordnung eines staats- und eurozentristischen Kriegsverständnisses (etwa Daase 2011; Geis 2006 b; Schlichte 2006) analytisches Potenzial, das Agieren externer Akteur_innen in Konfliktanalysen miteinzubeziehen (etwa Greven 2013: 90; König 2013; Shuriye/Ibrahim 2013: 511-512). Der besondere Stellenwert postkolonialer Kritikperspektiven für die konflikttheoretische Deutung des Wandels des globalen Kriegsgeschehens ergibt sich dann dadurch, die Wirkungsmächtigkeit unabgeschlossener Kolonialpraktiken in Verschränkung diskursiver Repräsentationsformen und materieller Herrschaftsverhältnisse zu konzeptualisieren. Konflikttheoretisch wären bei der Suche nach Ursachen und Dynamiken für den Wandel der Kriegsformen dann nicht nur die vielfältigen materiellstrukturellen Bedingungen von Herrschaft oder Ökonomie zu untersuchen, sondern auch zu fragen, wie Konflikträume von innen und außen durch Diskurse und politisches (Nicht-)Handeln konstruiert werden (bzw. wie dem Materiellen durch

34 Diese Verweise beziehen sich auf empirische Konfliktanalysen zu den gewaltsamen Auseinandersetzungen in Mali 2012. 
soziale Akteur_innen Sinn zugeschrieben wird) und wie sich durch das vielfältige Konflikthandeln entlang unterschiedlicher Ebenen die Bedingungen und Räume der Gewalt selbst verändern. Hinsichtlich der Suche nach theoretisch geleiteten Erklärungen für die Dynamiken und den Wandel des Krieges wären dabei insbesondere die Verwobenheit lokaler Gewaltformationen mit transnationalen Konfliktdynamiken, globalen Machtverhältnissen und daraus resultierenden externen Interventionspraktiken zu reflektieren. Gleichzeitig bietet sich aber auch eine analytische Perspektive an, die »friedenspolitischen Interventionspraktiken « genauer zu dechiffrieren und auf ihren gewaltursächlichen oder kriegsverlängernden Gehalt hin zu untersuchen - und dabei auch zu hinterfragen, inwieweit »westliche« Staaten durch ihre diskursiven Praktiken der Verknüpfung terroristischer Gefährdungen, fragiler Staatlichkeit und »neuer Kriege« zu einem komplexen Bedrohungsszenario einerseits und andererseits infolge ihres gewandelten (und gescheiterten) militärischen Interventionshandelns selbst zu Veränderungen der empirischen Wirklichkeiten in Kriegen als auch zum Wandel des Krieges (im Sinne unserer wissenschaftlichen Vorstellungen davon) beitragen.

\section{Literatur}

Ahlbrecht, Kathrin/Bendiek, Annegret/Meyers, Reinhard/Wagner, Sabine 2009: Konfliktregelung und Friedenssicherung im internationalen System, Wiesbaden. Arndt, Susan 2011: Rasse, in: Arndt, Susan/Ofuatey-Alazard, Nadja (Hrsg.): Wie Rassismus aus Wörtern spricht. (K)Erben des Kolonialismus im Wissensarchiv deutscher Sprache, Münster, 660-664.

Ayers, Alison J. 2006: Beyond the Imperial Narrative. African Political Historiography Revisited, in: Gruffyd Jones, Branwen (Hrsg.): Decolonizing International Relations, Lanham, MD, 155-177.

Azam, Jean-Paul 2006: On Thugs and Heroes. Why Warlords Victimize Their Own Civilians, in: Economics of Governance 7: 1, 53-73.

Baberowski, Jörg 2007: Kriege in staatsfernen Räumen: Rußland und die Sowjetunion 1905-1950, in: Beyrau, Dietrich/Hochgeschwender, Michael/Langewiesche, Dieter (Hrsg.): Formen des Krieges. Von der Antike bis zur Gegenwart, Paderborn, 291-309.

Baines, Erin 2003: Rwanda and the Politics of the Body (Working Paper No. 39, Centre of International Relations, University of British Columbia), Vancouver. Belina, Bernd 2013: Raum. Zu den Grundlagen eines historisch-geographischen Materialismus, Münster. 
Belina, Bernd/Miggelbrink, Judith 2010: Hier so, dort anders. Zum Vergleich von Raumeinheiten in der Wissenschaft und anderswo, in: dies. (Hrsg.): Hier so, dort anders. Raumbezogene Vergleiche in der Wissenschaft und anderswo, Münster, 7-39.

Benseler, Frank/Blanck, Bettina/Keil, Reinhard/Loh, Werner (Hrsg.) 2008: Erwägen Wissen Ethik 19: 1, Paderborn.

Berdal, Mats 2011: The `New Wars`Thesis Revisited, in: Strachan, Hew/Scheipers, Sibylle (Hrsg.): The Changing Character of War, Oxford, 109-133.

Bergmann, Anna 2010: Gewalt und Männlichkeit. Wahrnehmungsmuster des >Fremden und des >Eigenen < in der deutschen Berichterstattung über den Afghanistankrieg, in: Thiele, Martina/Thomas, Tanja/Virchow, Fabian (Hrsg.): Medien - Krieg - Geschlecht. Affirmationen und Irritationen sozialer Ordnungen, Wiesbaden, 153-172.

Bialasiewicz, Luiza/Campbell, David/Elden, Stuart/Graham, Stephen/Jeffrey, Alex/ Williams Alison J. 2007: Performing Security. The Imaginative Geographies of Current US Strategy, in: Political Geography 26: 4, 405-422.

Bilgin, Pinar/Morton, Adam David 2002: Historicising Representations of $>$ Failed States : Beyond the Cold-War Annexation of the Social Sciences?, in: Third World Quarterly 23: 1, 55-80.

Blagojević, Marina 2004: Conflict, Gender and Identity. Conflict and Continuity in Serbia, in: Seifert, Ruth (Hrsg.): Gender, Identität und kriegerischer Konflikt. Das Beispiel des ehemaligen Jugoslawien, Münster, 68-88.

Blattman, Christopher/Miguel, Edward 2010: Civil War, in: Journal of Economic Literature 48: 1, 3-57.

Boemcken, Marc von/Werkner, Ines-Jacqueline/Johannsen, Margret/Schoch, Bruno 2013: Stellungnahme der Herausgeber und Herausgeberinnen. Aktuelle Entwicklungen und Empfehlungen, in: dies. (Hrsg.): Friedensgutachten 2013, Münster, 1-30.

Bonacker, Thorsten 2006: Krieg und Theorie der Weltgesellschaft. Zur makrosoziologischen Erklärung neuerer Ergebnisse der empirischen Kriegsforschung, in: Geis, Anna 2006 a, 75-94.

Bonacker, Thorsten/Imbusch, Peter 2010: Zentrale Begriffe der Friedens- und Konfliktforschung. Konflikt, Gewalt, Krieg, Frieden, in: Imbusch, Peter/Zoll, Ralf (Hrsg.): Friedens- und Konfliktforschung. Eine Einführung, 5. Auflage, Wiesbaden, 67-142.

Brock, Lothar 2000: Modernisierung und Entgrenzung. Zwei Perspektiven der Weltgesellschaft, in: Siegelberg, Jens/Schlichte, Klaus (Hrsg.): Strukturwandel internationaler Beziehungen, Opladen, 281-303. 
Brownmiller, Susan 1975: Against Our Will. Men, Women and Rape, New York, NY.

Brunner, Claudia 2011: Wissensobjekt Selbstmordattentat. Epistemische Gewalt und okzidentalische Selbstvergewisserung in der Terrorismusforschung, Wiesbaden.

Brunner, Claudia/Hrzán, Daniela 2009: Female Suicide Bombing - Female Genital Cutting. Wissen über »die ganz Andere« im Spannungsfeld von physischer, politischer und epistemischer Gewalt, in: Femina Politica 18: 2, 95-105.

Buhaug, Halvard 2010: Dude, Where's My Conflict? LSG, Relative Strength, and the Location of Civil War, in: Conflict Management and Peace Science 27: 2, 107-128.

Bundeszentrale für politische Bildung 2007 a: Neues vom Chamäleon Krieg Essay, in: http://www.bpb.de/apuz/30520/neues-vom-chamaeleon-krieg-essay; 19.8.2014.

Bundeszentrale für politische Bildung 2007 b: »Ein Korsett für Schwache«. Interview mit Herfried Münkler, in: http://www.bpb.de/politik/extremismus/islamis mus/36385/interview-muenkler; 19.8.2014.

Bundeszentrale für politische Bildung 2011 a: 10 Jahre 9/11 - Terror hoch 10?, in: http://www.bpb.de/mediathek/44033/10-jahre-9-11-terror-hoch-10; 19.8.2014. Bundeszentrale für politische Bildung 2011 b: Was ist Krieg?, in: http://www.bpb. $\mathrm{de} /$ gesellschaft/medien/krieg-in-den-medien/130595/interview-mit-herfriedmuenkler; 19.8.2014.

Butler, Judith 2009: Raster des Krieges. Warum wir nicht jedes Leid beklagen, Frankfurt a. M.

Castro Varela, María Do Mar/Dhawan, Nikita 2005: Postkoloniale Theorie. Eine kritische Einführung, Bielefeld.

Castro Varela, María Do Mar/Dhawan, Nikita 2009: Europa provinzialisieren? Ja, bitte! Aber wie?, in: Femina Politica 18: 2, 9-18.

Chakrabarty, Dipesh 2002: Europa provinzialisieren. Postkolonialität und die Kritik der Geschichte, in: Conrad, Sebastian/Randeria, Shalini (Hrsg.): Jenseits des Eurozentrismus. Postkoloniale Perspektiven in den Geschichts- und Kulturwissenschaften, Frankfurt a. M., 283-312.

Chojnacki, Sven 2004: Wandel der Kriegsformen? Ein kritischer Literaturbericht, in: Leviathan 32: 3, 402-424.

Chojnacki, Sven 2006: Kriege im Wandel. Eine typologische und empirische Bestandsaufnahme, in: Geis, Anna 2006 a, 47-74. 
Chojnacki, Sven 2007: Auf der Suche nach des Pudels Kern: Alte und Neue Typologien in der Kriegsforschung, in: Beyrau, Dietrich/Hochgeschwender, Michael/ Langewiesche, Dieter (Hrsg.): Formen des Krieges. Von der Antike bis zur Gegenwart, Paderborn, 479-502.

Cline, Lawrence E. 2013: Nomads, Islamists, and Soldiers. The Struggles for Northern Mali, in: Studies in Conflict and Terrorism 36: 8, 617-634.

Collier, Paul/Hoeffler, Anke 2004: Greed and Grievance in Civil War, in: Oxford Economic Papers 56: 4, 563-595.

Conrad, Sebastian/Randeria, Shalini 2002: Geteilte Geschichten. Europa in einer postkolonialen Welt, in: dies. (Hrsg.): Jenseits des Eurozentrismus. Postkoloniale Perspektiven in den Geschichts- und Kulturwissenschaften, Frankfurt a. M., 9-49.

Creveld, Martin van 1991: The Transformation of War. The Most Radical Reinterpretation of Armed Conflict since Clausewitz, New York, NY.

Daase, Christopher 2006: Die Theorie des Kleinen Krieges revisited, in: Geis, Anna 2006 a, 151-166.

Daase, Christopher 2011: Neue Kriege und neue Kriegführung als Herausforderungen für die Friedenspolitik, in: Werkner, Ines-Jacqueline/Kronfeld-Goharani, Ulrike (Hrsg.): Der ambivalente Frieden. Die Friedensforschung vor neuen Herausforderungen, Wiesbaden, 21-35.

Daxner, Michael 2010: Das Konzept von Interventionskultur als Bestandteil einer gesellschaftsorientierten theoretischen Praxis, in: Bonacker, Thorsten/Daxner, Michael/Free, Jan H./Zürcher, Christoph (Hrsg.): Interventionskultur. Zur Soziologie von Interventionsgesellschaften, Wiesbaden, 75-100.

Dexter, Helen 2007: New War, Good War and the War on Terror. Explaining, Excusing and Creating Western Neo-Interventionism, in: Development and Change 38: 6, 1055-1071.

Dexter, Helen 2008: The ^New War War< Revival, in: Government and Opposition 43: 1, 55-78.

Dhawan, Nikita 2009: Zwischen Empire und Empower. Dekolonisierung und Demokratisierung, in: Femina Politica 18: 2, 52-63.

Eck, Kristine/Hultman, Lisa 2007: One-Sided Violence against Civilians in War. Insights from New Fatality Data, in: Journal of Peace Research 44: 2, 233-246.

Ehrhart, Hans-Georg 2013: Mali. Auf dem Weg zu einem »Sahelistan«?, in: Boemcken, Marc von/Werkner, Ines-Jacqueline/Johannsen, Margret/Schoch, Bruno (Hrsg.): Friedensgutachten 2013, Berlin, 251-263. 
Elwert, Georg 1997: Gewaltmärkte. Beobachtungen zur Zweckrationalität der Gewalt, in: Trotha, Trutz von (Hrsg.): Soziologie der Gewalt: Kölner Zeitschrift für Soziologie und Sozialpsychologie, Sonderheft 37/1997, 86-101.

Engels, Bettina 2014: Repräsentationen, Diskurse und Machtfragen: Postkoloniale Theorieansätze in der Friedens- und Konfliktforschung, in: Zeitschrift für Friedens- und Konfliktforschung 3: 1, 127-146.

Exo, Mechthild 2009: Demokratie von unten, Demokratie von oben, in: http:// www.akweb.de/ak_s/ak540/36.htm; 21.8.2014.

Exo, Mechthild 2011: You do not hear us! Die unbekannte linke und feministische Opposition in Afghanistan, in: http://www.akweb.de/ak_s/ak563/13.htm; 21.8.2014.

Fanon, Frantz 1981: Die Verdammten dieser Erde, Frankfurt a. M.

Feierman, Steven 2002: Afrika in der Geschichte. Das Ende der universalen Erzählungen, in: Conrad, Sebastian/Randeria, Shalini (Hrsg.): Jenseits des Eurozentrismus. Postkoloniale Perspektiven in den Geschichts- und Kulturwissenschaften, Frankfurt a. M., 50-83.

Frech, Siegfried/Trummer, Peter I. 2005: Neue Kriege. Akteure, Gewaltmärkte, Ökonomie, Frankfurt a. M.

Gärtner, Heinz 2008: Antwort auf Münklers »Krieg«: Kriegsdefinition zu weit gefasst!, in: Erwägen Wissen Ethik 19: 1, 64-65.

Gantzel, Klaus Jürgen 2002: Neue Kriege? Neue Kämpfer? (Arbeitspapier Nr. 2, Forschungsstelle Kriege, Rüstung und Entwicklung), Hamburg.

Gareis, Sven Bernhard 2012: Länder im Chaos (Y-Magazin, 14.8.2012), in: http:// bit.ly/1tAAx1z; 26.8.2014.

Garfinkel, Michelle R./Skaperdas, Stergios 2006: Economics of Conflict. An Overview, in: Sandler, Todd/Hartley, Keith (Hrsg.): Handbook of Defense Economics, 2. Auflage, New York, NY, 649-709.

Geis, Anna (Hrsg.) 2006 a: Den Krieg überdenken. Kriegsbegriffe und Kriegstheorien in der Kontroverse, Baden-Baden.

Geis, Anna 2006 b: Den Krieg überdenken. Kriegsbegriffe und Kriegstheorien in der Kontroverse, in: dies. 2006 a, 9-46.

Goldstone, Jack A. 2008: Pathways to State Failure, in: Conflict Management and Peace Science 25: 4, 285-296.

Gregory, Derek 2010: War and Peace, in: Transactions of the Institute of British Geographers 35: 2, 154-186.

Greven, Thomas 2013: Tuareg-Rebellion, Islamismus und Staatskrise in Mali, in: PROKLA 43: 1, 89-96. 
Grovogui, Siba N'Zatioula 2006: Mind, Body, and Gut! Elements of a Postcolonial Human Rights Discourse, in: Gruffyd Jones, Branwen (Hrsg.): Decolonizing International Relations, Lanham, MD, 179-196.

Henderson, Errol A./Singer, J. David 2002: »New Wars« and Rumors of »New Wars «, International Interactions. Empirical and Theoretical Research in International Relations 28: 2, 165-190.

Heupel, Monika/Zangl, Bernhard 2004: Von »alten« und »neuen« Kriegen. Zum Gestaltwandel kriegerischer Gewalt, in: Politische Vierteljahresschrift 45: 3, 346-369.

Hill, Jonathan 2005: Beyond the Other? A Postcolonial Critique of the Failed State Thesis, in: African Identities 3: 2, 139-154.

Hunt, Krista 2006: >Embedded Feminism < and the War on Terror, in: Hunt, Krista/ Rygiel, Kim (Hrsg.): (En)Gendering the War on Terror. War Stories and Camouflaged Politics, Aldershot, 51-71.

Jahn, Egbert 2012: Frieden und Konflikt, Wiesbaden.

Jellinek, Georg 1900: Allgemeine Staatslehre, Berlin.

Jessop, Bob/Brenner, Neil/Jones, Martin 2008: Theorizing Sociospatial Relations, in: Environment and Planning D: Society and Space 26: 3, 389-401.

Kahl, Martin/Teusch, Ulrich 2004: Sind die »neuen Kriege« wirklich neu?, in: Leviathan 32: 3, 382-401.

Kaldor, Mary 1999: New and Old Wars. Organized Violence in a Global Era, Cambridge.

Kaldor, Mary 2001: New Types of Conflict, in: Stanley, Ruth (Hrsg.): Gewalt und Konflikt in einer globalisierten Welt, Wiesbaden, 24-50.

Kaldor, Mary 2012: New and Old Wars. Organized Violence in a Global Era, 3. Auflage, Cambridge.

Kaldor, Mary 2013: In Defence of New Wars, in: Stability 2: 1, 1-16.

Kalyvas, Stathis N. 2001: »New« And »Old« Civil Wars. A Valid Distinction?, in:

World Politics 54: 1, 99-118.

Kalyvas, Stathis N. 2006: The Logic of Violence in Civil War, New York, NY.

Kennedy, Caroline/Waldman, Thomas 2014: The Changing Nature of Intrastate Conflict and `New Wars`, in: Newman, Edward/DeRouen, Karl Jr. (Hrsg.): Routledge Handbook of Civil Wars, London, 213-223.

Kilomba, Grada 2008: Plantation Memories. Episodes of Everyday Racism, 2. Auflage, Münster. 
Klaus, Elisabeth/Kassel, Susanne 2008: Frauenrechte als Kriegslegitimation in den Medien, in: Dorer, Johanna/Geiger, Brigitte/Köpl, Regina (Hrsg.): Medien Politik - Geschlecht. Feministische Befunde zur politischen Kommunikationsforschung, Wiesbaden, 266-280.

König, Claus-Dieter 2013: Mali: Vom »Hoffnungsträger« zum »Krisenstaat«. Auf der Suche nach Lösungen jenseits von militärischem Interventionismus, in: http://www.rosalux.de/fileadmin/rls_uploads/pdfs/Standpunkte/Standpunkte_ international/Standpunkte_int_06-2013.pdf; 19.6.2014.

Koubi, Vally/Spilker, Gabriele/Böhmelt, Tobias/Bernauer, Thomas 2013: Do Natural Resources Matter for Interstate and Intrastate Armed Conflict?, in: Journal of Peace Research 50: 5, 1-17.

Krell, Gert 2009: Weltbilder und Weltordnung. Einführung in die Theorie der internationalen Beziehungen, 4. Auflage, Baden-Baden.

Krippendorff, Ekkehart 1977: Internationale Beziehungen als Wissenschaft, Frankfurt a. M.

Krippendorff, Ekkehart 1985: Staat und Krieg. Die historische Logik politischer Unvernunft, Frankfurt a. M.

Krippendorff, Ekkehart 1986: Internationale Politik. Geschichte und Theorie, Frankfurt a. M.

Le Billon, Phillipe 2007: Geographies of War. Perspectives on 〉Resource Wars`, in: Geography Compass 1: 2, 163-182.

Lecocq, Baz/Mann, Gregory/Whitehouse, Bruce/Badi, Dida/Pelckmans, Lotte/ Belalimat, Nadia/Hall, Bruce/Lacher, Wolfram 2013 a: Ein Flusspferd und acht blinde Analysten, in: inamo 19: 75, 16-22.

Lecocq, Baz/Mann, Gregory/Whitehouse, Bruce/Badi, Dida/Pelckmans, Lotte/ Belalimat, Nadia/Hall, Bruce/Lacher, Wolfram 2013 b: One Hippopotamus and Eight Blind Analysts. A Multivocal Analysis of the 2012 Political Crisis in the Divided Republic of Mali, in: Review of African Political Economy 40:3, 343-357.

L'Express 2013: Hollande répond à Sarkozy sur le Mali, in: http://www.lexpress. fr/actualite/politique/hollande-la-france-est-au-mali-parce-qu-il-y-avait-des-fe mmes-victimes-de-l-oppression-et-de-la-barbarie_1228297.html; 21.8.2014.

List, Martin 2006: Internationale Politik studieren. Eine Einführung, Wiesbaden.

Lounnas, Djallil 2013: The Regional Fallouts of the French Intervention in Mali, in: Mediterranean Politics 18: 2, 325-332.

Maier, Tanja/Balz, Hanno 2010: Orientierungen. Bilder des $>$ Fremden $<$ in medialen Darstellungen von `Krieg und Terror`, in: Thiele, Martina/Thomas, Tanja/Virchow, Fabian (Hrsg.): Medien - Krieg - Geschlecht. Affirmationen und Irritationen sozialer Ordnungen, Wiesbaden, 81-101. 
Malešević, Siniša/Dochartaigh, Niall Ó. 2014: Sociological Approaches, in: Newman, Edward/DeRouen, Karl Jr. (Hrsg.): Routledge Handbook of Civil Wars, London, 54-66.

Matthies, Volker 2004: Eine Welt voller neuer Kriege, in: Der Bürger im Staat 54: 4, 185-190.

Melander, Eric/Öberg, Magnus/Hall, Jonathan 2009: Are `New Wars〈 More Atrocious? Battle Severity, Civilians Killed and Forced Migration Before and After the End of the Cold War, in: European Journal of International Relations 15: 3, 505-536.

Meyers, Reinhard 2011 a: Krieg und Frieden, in: Woyke, Wichard (Hrsg.): Handwörterbuch Internationale Politik, 12. Auflage, Bonn, 302-323.

Meyers, Reinhard 2011 b: Krieg und Frieden, in: Gießmann, Hans J./Rinke, Bernhard (Hrsg.): Handbuch Frieden, Wiesbaden, 21-52.

Mielke, Katja/Schetter, Conrad/Wilde, Andreas 2011: Dimensions of Social Order. Empirical Fact, Analytical Framework and Boundary Concept (ZEF Working Paper Series 78), Bonn.

Münkler, Herfried 2002: Die neuen Kriege, Berlin.

Münkler, Herfried 2008: Krieg, in: Erwägen Wissen Ethik 19: 1, 27-43.

Münkler, Herfried 2010: Der Wandel des Krieges. Von der Symmetrie zur Asymmetrie, Göttingen.

Münkler, Herfried 2011: Neue Kriege, in: Nohlen, Dieter/Grotz, Florian (Hrsg.): Kleines Lexikon der Politik, 5. Auflage, München, 393-394.

Münkler, Herfried 2013: Von der konventionellen Kriegführung zur Abwehr asymmetrischer Gewalt. Zur Theorie der »neuen Kriege«, in: Bohrmann, Thomas/ Lather, Karl-Heinz/Lohmann, Friedrich (Hrsg.): Handbuch militärische Berufsethik, Wiesbaden, 253-272.

Münkler, Herfried 2014 a: Die gefährliche Kluft zwischen Schein und Tun, in: http:// www.review2014.de/de/pdf/aussensicht/show/article/die-gefaehrliche-kluftzwischen-schein-und-tun.html; 19.8.2014.

Münkler, Herfried 2014 b: Politologe Münkler zum Gauck-Streit: »Ach, die Stechschrittpazifisten von der Linken «, in: http://www.spiegel.de/politik/deutschland/ gauck-streit-um-bundeswehr-muenkler-unterstuetzt-bundespraesident-a-97835 2.html; 18.8.2014.

Newman, Edward 2004: The $>$ New Wars $<$ Debate. A Historical Perspective is Needed, in: Security Dialogue 35: 2, 173-189.

Newman, David 2006: The Resilience of Territorial Conflict in an Era of Globalization, Beer Sheva, unv. Ms. 
Oberschall, Anthony 2000: The Manipulation of Ethnicity. From Ethnic Cooperation to Violence and War in Yugoslavia, in: Ethnic and Racial Studies, 23: 6, 982-1001.

Ofuatey-Alazard, Nadja 2011: Koloniale Kontinuitäten in Deutschland, in: Arndt, Susan/Ofuatey-Alazard, Nadja (Hrsg.): Wie Rassismus aus Wörtern spricht. (K)Erben des Kolonialismus im Wissensarchiv deutscher Sprache, Münster, 136-153.

Osterhammel, Jürgen 2003: Kolonialismus. Geschichte - Formen - Folgen, München.

Pabst, Martin 2013: Staatliche Schwäche begünstigt Dschihadisten. Neuer Risikoraum Sahelzone, in: Sicherheit und Frieden 31: 1, 1-57.

Plumelle-Uribe, Rosa Amelia 2004: Weiße Barbarei. Vom Kolonialrassismus zur Rassenpolitik der Nazis, Zürich.

Pöhlmann, Markus 2006: Die Rückkehr der Condottieri? Krieg und Militär im Spannungsfeld zwischen Verstaatlichung und Privatisierung. Die Entwicklung vom 16. Jahrhundert bis zur Gegenwart (Tagungsbericht), in: http://hsozkult. geschichte.hu-berlin.de/tagungsberichte/id=1146; 24.5.2014.

Randeria, Shalini 1999: Geteilte Geschichte und verwobene Moderne, in: Rüsen, Jörn/Leitgeb, Hannah/Jegelka, Norbert (Hrsg.): Zukunftsentwürfe. Ideen für eine Kultur der Veränderung, Frankfurt a. M., 87-96.

Randeria, Shalini 2009: Transnationalisierung des Rechts und der »listige Staat « in Indien. Zivilgesellschaftlicher Widerstand gegen die Privatisierung öffentlicher Güter, in: Randeria, Shalini/Eckert, Andreas (Hrsg.): Vom Imperialismus zum Empire. Nicht-westliche Perspektiven auf Globalisierung, Frankfurt a. M., 211-236.

Rasler, Karen/Thompson, William R. 2011: Borders, Rivalry, Democracy, and Conflict in the European Region, 1816-1994, in: Conflict Management and Peace Science 28: 3, 280-305.

Reno, William 2011: Warfare in Independent Africa. New Approaches to African History, Cambridge.

Revolutionary Association of the Women of Afghanistan 2008: Neither the US nor Jehadies and Taliban. Long Live the Struggle of Independent and Democratic Forces of Afghanistan!, in: http://www.rawa.org/events/sevenyear_e.htm; 21.8.2014.

Reyna, Stephen 2008: Global Warring Today: »Maybe Somebody Needs to Explain «, in: Social Analysis 52: 2, 50-70.

Reyna, Stephen 2009: Taking Place: `New Wars $<$ Versus Global Wars, in: Social Anthropology/Anthropologie Sociale 17: 3, 291-317. 
Ross, Michael L. 2004: What Do We Know about Natural Resources and Civil War?, in: Journal of Peace Research 41: 3, 337-356.

Rotberg, Robert I. 2004: The Failure and Collapse of Nation-States. Breakdown, Prevention, and Repair, in: ders. (Hrsg.): When States Fail. Causes and Consequences, Princeton, NJ, 1-50.

Rotberg, Robert I. 2007: The Challenge of Weak, Failed, and Collapsed States, in: Crocker, Chester A./Hampson, Fen Osler/Aall, Pamela (Hrsg.): Leashing the Dogs of War. Conflict Management in a Divided World, Washington, D.C., 83-94.

Sahm, Astrid/Sapper, Manfred/Weichsel, Volker 2006: Frieden und Krieg in Zeiten des Übergangs, in: dies. (Hrsg.): Die Zukunft des Friedens. Band 1: Eine Bilanz der Friedens- und Konfliktforschung, 2. Auflage, Wiesbaden, 9-18.

Said, Edward 2009: Orientalismus, 4. Auflage, Frankfurt a. M.

Sarkees, Meredith 2014: Patterns of Civil Wars in the Twenty-First Century. The Decline of Civil War?, in: Newman, Edward/DeRouen, Karl Jr. (Hrsg.): Routledge Handbook of Civil Wars, London, 236-257.

Schlichte, Klaus 2006: Neue Kriege oder alte Thesen? Wirklichkeit und Repräsentation kriegerischer Gewalt in der Politikwissenschaft, in: Geis, Anna 2006 a, 111-132.

Schneckener, Ulrich 2004: States at Risk. Zur Analyse fragiler Staatlichkeit, in: ders. (Hrsg.): States at Risk. Fragile Staaten als Sicherheits- und Entwicklungsproblem, Berlin, 5-27.

Schneckener, Ulrich 2005: Fragile Staatlichkeit als globales Sicherheitsrisiko, in: http://www.bpb.de/apuz/28954/fragile-staatlichkeit-als-globales-sicherheitsrisiko? $\mathrm{p}=$ all; 27.8.2014.

Seifert, Ruth 2002: Identität, Militär und Geschlecht. Zur identitätspolitischen Bedeutung einer kulturellen Konstruktion, in: Hagemann, Karin/Schüler-Springorum, Stefanie (Hrsg.): Heimat-Front. Militär und Geschlechterverhältnisse im Zeitalter der Weltkriege, Frankfurt a. M., 53-66.

Shuriye, Abdi O./Ibrahim, Dauda Sh. 2013: The Role of Islam and Natural Resources in Current Mali Political Turmoil, in: Mediterranean Journal of Social Sciences 4: 6, 507-513.

Smith, Neil 1987: Dangers of the Empirical Turn. Some Comments on the CURS Initiative, in: Antipode 19: 1, 59-68.

Smith, Neil 1990: Afterword. The Beginning of Geography, in ders. (Hrsg.): Uneven

Development. Nature, Capital, and the Production of Space, 2. Auflage, Oxford, 160-178. 
Sow, Noah 2011: Ist deutscher Rassismus Geschichte?, in: Arndt, Susan/OfuateyAlazard, Nadja (Hrsg.): Wie Rassismus aus Wörtern spricht. (K)Erben des Kolonialismus im Wissensarchiv deutscher Sprache, Münster, 126-135.

Spivak, Gayatri Chakravorty 2008: Can the Subaltern Speak? Postkolonialität und subalterne Artikulation, Wien.

Strüver, Anke 2010: Geopolitische Diskurse nach 9/11 und der Krieg gegen Afghanistan in den deutschen Printmedien, in: Glunz, Claudia/Schneider, Thomas F. (Hrsg.): Wahrheitsmaschinen. Der Einfluss technischer Innovationen auf die Darstellung und das Bild des Krieges in den Medien und Künsten, Göttingen, 79-94.

Themnér, Lotta/Wallensteen, Peter 2013: Armed Conflicts, 1946-2012, in: Journal of Peace Research 50: 4, 509-521.

Thiele, Martina 2010: Medial vermittelte Vorurteile, Stereotype und $>$ Feindinnenbilder, in: Thiele, Martina/Thomas, Tanja/Virchow, Fabian (Hrsg.): Medien Krieg - Geschlecht. Affirmationen und Irritationen sozialer Ordnungen, Wiesbaden, 61-79.

Thomas, Tanja/Virchow, Fabian 2010: `Suicidal Attacks` und ihre medialen Repräsentationen. Geschlechtertheoretische Überlegungen und Befunde, in: Thiele, Martina/Thomas, Tanja/Virchow, Fabian (Hrsg.): Medien - Krieg Geschlecht. Affirmationen und Irritationen sozialer Ordnungen, Wiesbaden, 235-256.

Tilly, Charles 2003: The Politics of Collective Violence, Cambridge.

Traoré, Aminata D. 2013: Das Scheitern und der Affront. Mali ist den Maliern zurückzugeben, in: http://www.africavenir.org/de/news-archiv/newsdetails/ datum/2013/04/20/aminata-traore-le-naufrage-et-loffense-le-mali-est-a-rendreau-maliens.html; 21.8.2014.

Trouillot, Michel-Rolph 2002: Undenkbare Geschichte. Zur Bagatellisierung der haitischen Revolution, in: Conrad, Sebastian/Randeria, Shalini (Hrsg.): Jenseits des Eurozentrismus. Postkoloniale Perspektiven in den Geschichts- und Kulturwissenschaften, Frankfurt a. M., 84-115.

Veit, Alex 2008: Jenseits der Schuldfrage. Die politische Ökonomie des Krieges am Beispiel der DR Kongo, in: iz3w Nr. 304, 21-23.

Wai, Zubairu 2012: Neo-Patrimonialism and the Discourse of State Failure in Africa, in: Review of African Political Economy 39: 1, 27-43.

Weber, Max 1988: Politik als Beruf, in: Gesammelte Politische Schriften. Herausgegeben von Johannes Winckelmann, 5. Auflage, Tübingen, 505-560.

Weinstein, Jeremy M. 2007: Inside Rebellion. The Politics of Insurgent Violence, Cambridge. 
Weiss, Stefani/Schmierer, Joscha (Hrsg.) 2007: Prekäre Staatlichkeit und internationale Ordnung, Wiesbaden.

Winter, Yves 2011: The Asymmetric War Discourse and Its Moral Economies. A Critique, in: International Theory 3: 3, 488-514.

Wissen, Markus 2008: Zur räumlichen Dimensionierung sozialer Prozesse. Die Scale-Debatte in der angloamerikanischen Radical Geography - eine Einleitung, in: Wissen, Markus/Röttger, Bernd/Heeg, Susanne (Hrsg.): Politics of Scale. Räume der Globalisierung und Perspektiven emanzipatorischer Politik, Münster, 8-33.

Wollrad, Eske 2005: Weißsein im Widerspruch. Feministische Perspektiven auf Rassismus, Kultur und Religion, Königstein.

Wood, Barbara 2008: The Social Processes of Civil War. The Wartime Transformation of Social Networks, in: Annual Review of Political Science Vol. 11, 539-561.

Wood, Elisabeth 2006: Variation in Sexual Violence during War, in: Politics \& Society 34: 3, 307-342.

Young, Iris Marion 2003: The Logic of Masculinist Protection. Reflections on the Current Security State, in: Signs 29: 1, 1-25.

Zangl, Bernhard/Zürn, Michael 2003: Frieden und Krieg. Sicherheit in der nationalen und postnationalen Konstellation, Frankfurt a. M.

Zarkov, Dubravka 2001: The Body of the Other Man. Sexual Violence and the Construction of Masculinity, Sexuality and Ethnicity in the Croatian Media, in: Moser, Caroline O. N./Clark, Fiona C. (Hrsg.): Victims, Perpetrators or Actors? Gender, Armed Conflict and Political Violence, London, 69-82.

Ziai, Aram 2010: Postkoloniale Perspektiven auf »Entwicklung«, in: Peripherie 30: 4, 399-426.

Ziai, Aram 2012 a: Postkoloniale Politikwissenschaft. Grundlagen einer postkolonialen politischen Theorie und deren Anwendungsfelder, in: Karentzos, Alexandra/Reuter, Julia (Hrsg.): Schlüsselwerke der Postcolonial Studies, Wiesbaden, 283-296.

Ziai, Aram 2012 b: Postkoloniale Studien und Politikwissenschaft. Komplementäre Defizite, Stand der Forschung und Perspektiven, in: Politische Vierteljahresschrift 53: 2, 291-322. 


\section{Die Autoren}

Sven Chojnacki ist Professor für Vergleichende Politikwissenschaft und empirische Konfliktforschung und Leiter des Arbeitsschwerpunkts Friedens- und Konfliktforschung des Otto-SuhrInstituts für Politikwissenschaft, Freie Universität Berlin.

E-Mail: sven.chojnacki@fu-berlin.de

Fabian Namberger studierte Politikwissenschaft (B. A.) an der Freien Universität Berlin und absolviert derzeit ein Masterstudium am Goldsmiths College in London. Von 2013 bis 2014 war er als studentischer Mitarbeiter am Arbeitsschwerpunkt für Friedens- und Konfliktforschung des Otto-Suhr-Instituts für Politikwissenschaft, Freie Universität Berlin, tätig.

E-Mail: fnamb001@gold.ac.uk 